\title{
Maintenance of AMPK activation by metformin is beneficial for suppressing the progress of diabetes- accelerated atherosclerosis via inhibition of vascular smooth muscle cells migration
}

Yi Yan

The Third Affiliated Hospital of Guangzhou Medical University

\section{Ting Li}

The Third Affiliated Hospital of Guangzhou Medical University

Zhonghao Li

Southern Medical University

Mingyuan $\mathrm{He}$

The Third Affiliated Hospital of Guangzhou Medical University

Dejiang Wang

The Third Affiliated Hospital of Guangzhou Medical University

\section{Yingyi Xu}

The Third Affiliated Hospital of Guangzhou Medical University

Xuewen Yang

The Third Affiliate Hospital of Guangzhou Medical University

Yuanyuan Bai

Guangzhou First People's Hospital

Yi Lao

Zhong Shan Hospital of Sun Yet-sun University

Zhiyong Zhang

The Third Affiliated Hospital of Guangzhou Medical University

Wei Wu ( $\nabla$ wuwei2668@smu.edu.cn )

Southern Medical University

Original investigation

Keywords: atherosclerosis, vascular smooth muscle cells, metformin, AMPK, Pdlim5

Posted Date: November 18th, 2020

DOI: https://doi.org/10.21203/rs.3.rs-109664/v1 
License: (c) (i) This work is licensed under a Creative Commons Attribution 4.0 International License. Read Full License 


\section{Abstract}

Background: Our previous work revealed that augmented AMPK activation inhibit cell migration by phosphorylating its substrate Pdlim5. As medial VSMCs contribute to the major composition of atherosclerotic plaques, a hypothesis is raised that modulation of AMPK-Pdlim5 signal pathway could retard the development of atherosclerosis through inhibiting migration of VSMCs. Therefore, we initiate the present study to investigate whether AMPK agonist like metformin is beneficial for suppressing diabetes-accelerated atherosclerosis in a diabetic mouse model induced by streptozotocin and high fat diet.

Methods: For cell experiment, vascular smooth muscle cells (VSMCs) were overexpressed flag fused Pdlim5 and Pdlim5 mutant. Then the engineered VSMCs were introduced with metformin or control drug before determination of phosphorylated Pdlim5 with immunoblotting. For animal work, 8-week-old male $\mathrm{Apo} \mathrm{E}^{-/-}$mice were induced diabetes with streptozotocin and then were randomly divided into 8 groups: control group, metformin hydrochloride ( $300 \mathrm{mg} / \mathrm{kg} /$ day) group, wildtype-Pdlim5 (Pdlim5 WT) carried adenovirus (Ad) group, Ad Pdlim5 WT and Met group, Ad Pdlim5 S177A group, Ad Pdlim5 S177A and Met group, Ad Pdlim5 S177D group, Ad Pdlim5 S177D and Met group. All mice were fed with high fat diet after virus infection. At the end, mice were sacrificed to observe atherosclerotic plaques and deposition of VSMCs in plaques. Moreover, 12-15-week-old Myh11-cre-EGFP male mice were accepted ligation of the left carotid artery and randomly divided into control and metformin treatment group. Finally, the injured vessel of Myh11-cre-EGFP mice were isolated to analyze the relationship between AMPK activation and neointima formation.

Results: It was found that AMPK directly phosphorylate Pdlim5 at Ser177 in vitro, and metformin, an AMPK agonist, could induce phosphorylation of Pdlim5 indirectly and inhibition of cell migration as a result. Exogenous expression of phosphomimetic S177D-Pdlim5 inhibits lamellipodia formation and migration in VSMCs. It was also demonstrated that VSMCs contribute to the major composition of injuryinduced neointimal lesions, while metformin could alleviate the occlusion of carotid artery in a wire-injury mice model. In order to investigate the function of AMPK-Pdlim5 pathway in the context of pathological condition, $\mathrm{ApoE}^{-/-}$male mice were divided randomly into control, streptozocin and high fat diet-induced diabetes mellitus, STZ + HFD together with metformin or Pdlim5 mutant carried adenovirus treatment groups. The results showed increased plasma lipids and aggravated vascular smooth muscle cells infiltration into the atherosclerotic lesion in diabetic mice compared with control mice. However, metformin alleviated diabetes-induced metabolic disorders and atherosclerosis, as well as decreased VSMCs infiltration in atherosclerotic plaques, while Pdlim5 phospho-abolished mutant carried adenovirus S177A-Pdlim5 undermine this protective function.

Conclusions: The activation of AMPK-Pdlim5 pathway by chemicals like Metformin could inhibit formation of migratory machine of VSMCs and alleviate the progress of atherosclerotic plaques in diabetic mice. The maintenance of AMPK activity is beneficial for suppressing diabetes-accelerated atherosclerosis or metabolic syndrome. 


\section{Background}

Diabetes mellitus accelerates atherosclerosis, with greater vascular inflammation, larger necrotic core, and more diffuse atherosclerosis in the coronary arteries ${ }^{1,2}$. Atherosclerosis is thought to be triggered by a regional vascular inflammation that induced by dyslipidemia, especially high LDL-cholesterol levels and high remnant lipoprotein levels ${ }^{3}$. The whole process could be summarized as follows: The circulating monocytes are attracted to the injured vascular endothelium and infiltrate the subendothelial space, where the monocytes differentiate into macrophages, which take up oxidized LDL cholesterol, and become foam cell finally ${ }^{4,5,6}$. The ox-LDL also induces the accumulation of vascular smooth muscle cells in the intima ${ }^{7}$. VSMCs are thought to be the major source of extracellular matrix (ECM) and foam cells in fatty streaks (pre-atherosclerotic plaques) ${ }^{3,8}$. With time, these early fatty streak lesions develop into advanced lesions, and some of which eventually become unstable and rupture, resulting in the adverse clinical events of $\mathrm{CVD}^{9,7,10}$. As described above, atherosclerotic lesions are formed through complex interactions of various factors, and insulin resistance and hyperglycemia in DM accelerates all these interactions, probably due to excessive and prolonged production of reactive oxidative species $(\mathrm{ROS})^{3,10}$. Many clinical trials showed that intensive glucose therapy in patients with type 2 diabetes mellitus (T2DM) reduce the risk of cardiovascular disease ${ }^{11,12}$. It is found that metformin, a oral hypoglycemic agent, exhibits abilities to suppress the progression of common carotid intima-media thickness in T2DM patients, and also reduce the incidence of myocardial infarction ${ }^{11,12,13,14}$. However, it is paradoxically that several recent clinical trials showed that the anti-atherogenic effect of metformin seems independent of its hypoglycemic function, because other regular therapies, such as insulin and sulfonylurea, possess less beneficial cardiovascular effects ${ }^{15,16,17,18}$. A possible target of metformin is AMP activated protein kinase (AMPK), a cellular energy sensor activated under metabolic stress ${ }^{19}$. The activation of AMPK inhibits hepatic glucose production, improves insulin sensitivity and glucose uptake by muscle, and induces fatty acid oxidation ${ }^{18}$. Our previous findings indicate that AMPK phosphorylate PDZ and LIM domain 5 (Pdlim5), a protein involved in cytoskeleton organization, on Ser177 to inhibit vascular smooth muscle cell migration by suppressing the Rac1-Arp2/3 signaling pathway ${ }^{20}$. As a major cell type present at all stages of an atherosclerotic plaque, VSMCs were used to be thought adverse at early atherosclerosis, because the migration of VSMCs from media to intima promotes plaque formation, but VSMC accumulation in advanced plaques was considered entirely beneficial for plaque stability due to their ability to generate ECM and form fibrous cap ${ }^{8}$. However, this view is now challenged by recent genetic lineage tracing studies, which reveal the existence of a subset of phenotype switched VSMC in the vessel wall during atherogenesis ${ }^{21}$. Those phenotype-switched VSMCs contribute to many cell components in the plaque, including the ECM-synthesizing cells, macrophage-like cells, foam cells, and etc. VSMCs also contribute to calcification and necrotic core after their apoptosis and necrosis, which are crucial to plaque instability ${ }^{8,22}$. The single-cell omics also reveals the heterogeneity of VSMCs in healthy vessel, which suggest the complexity of roles VSMCs played in the development of atherosclerosis ${ }^{23}$. Considering the importance of VSMCs in the atherosclerotic plaques, we assume that activation of AMPK-Pdlim5 pathway by metformin may be beneficial for suppressing diabetes accelerated 
atherosclerosis via inhibition of VSMCs migration. In this study, we identified that metformin could induce phosphorylation of Pdlim5 at Ser177 site through AMPK and inhibit cell migration in vitro. With vascular smooth muscle lineage tracking mice, we found that VSMCs from media contribute to the neointima formation after artery injury and metformin reduces VSMCs migration and area of neointima. Use streptozotocin (STZ)-induced diabetic $\mathrm{ApoE}^{-/-}$mice, we found that metformin reduces STZ and HFD induced atherosclerosis, while S177A-Pdlim5, an unphosphorylatable mutant, carried adenovirus undermine the Metformin's anti-atherosclerosis function. Taken together, metformin reduces the motility of vascular smooth muscle cells through activation of AMPK-Pdlim5 pathway, which contribute to the protective effects of metformin against diabetes accelerated atherosclerosis and beneficial for the therapy of metabolic syndrome.

\section{Methods}

\section{Animal experiments}

Eight-week-old male Apo $E^{-/-}$mice (body weight $20-25 \mathrm{~g}$ ) on C57/BL6 background were purchased from Beijing Biocytogen Co. Ltd (Beijing, China) and kept with free access to water and food in specific pathogen-free room under $24^{\circ} \mathrm{C}$ and 12 -h light/dark cycle at the laboratory animal center of Southern medical university. ApoE ${ }^{-/}$mice were injected intraperitoneally with $50 \mathrm{mg} / \mathrm{kg}$ streptozocin (STZ) for 5 days to induce DM. Two weeks later, Diabetic mice were randomly divided into 8 groups $(n=10$ per group): control group, metformin hydrochloride (Via gastric gavage, $300 \mathrm{mg} / \mathrm{kg} /$ day, Sigma-Aldrich) group, wildtype-Pdlim5 (Pdlim5 WT) carried adenovirus (Ad) group, Ad Pdlim5 WT and Met group, Ad Pdlim5 S177A group, Ad Pdlim5 S177A and Met group, Ad Pdlim5 S177D group, Ad Pdlim5 S177D and Met group. 3 days after virus infection, mice were fed with high fat diet subsequently. At the end of the experiments, mice were euthanized with terminal anaesthetic (isoflurane $>4 \%$ in $95 \% 025 \% \mathrm{CO} 2$ ). All animal experiments were approved and performed according to Institutional Animal Care and Use Committee (IACUC) of Southern Medical University, which conform to the guidelines from Directive 2010/63/EU of the European Parliament on the protection of animals used for scientific purposes. The high fat and high cholesterol diet were purchased from Guangdong Experimental Animal Center. The two $\mathrm{kg}$ per pack of HFD contains $4.4 \mathrm{kcal} / \mathrm{g}$ of energy, and the components per pack as listed by the manufacturer as follows: $17 \%$ lard, $1.2 \%$ cholesterol, $0.2 \%$ sodium cholate, $10 \%$ casein, $0.6 \%$ calcium hydrogencarbonate, $0.4 \%$ stone powder, $0.4 \%$ premix, $52.2 \%$ basic feed.

\section{Blood glucose and plasma lipids measurements}

Blood glucose level was determined 2 weeks later after STZ induction with OneTouch Ultra2 Glucose Monitors (LifeScan, Milpitas, CA, USA). Mice those blood glucose level above $16.6 \mathrm{mmol} / \mathrm{L}$ were diagnosed as having DM. Plasma total cholesterol (TC), triglyceride (TG), low-density lipoprotein cholesterol (LDL-C) and high-density lipoprotein cholesterol (HDL-C) were determined with biochemical kits (Jiancheng Biotechnology, Nanjing, China).

\section{Carotid artery injury}


12-15-week-old Myh11-cre-EGFP male mice background in C57BL/6 WT were purchased from Shanghai Model Organisms Center, Inc. The carotid arterial intima of mice were mechanically damaged with a beaded guidewire as reference described ${ }^{23}$. The mice were inhalationally anesthetized with mixture of isoflurane (2\%)-oxygen ( $98 \%$ ). The animal work was also approved and performed according to Institutional Animal Care and Use Committee (IACUC) of Southern Medical University, which conform to the guidelines from Directive 2010/63/EU of the European Parliament on the protection of animals used for scientific purposes.

\section{Tissue collection, en face analysis of the aortic arch, and Immunohistochemical staining}

The mice were perfused with phosphate buffer saline (PBS) followed by $4 \%$ paraformaldehyde after euthanasia. Hearts, together with a short segment of aorta, were collected, embedded or quick-frozen. Immunohistochemical staining was performed as described previously ${ }^{24}$. All immunofluorescence micrographs and oil red O-stained area of the atherosclerotic lesion were acquired with an Olympus FV1000 confocal laser scanning microscope (Olympus, Tokyo, Japan) and morphometric analysis by the Image J software $(\mathrm{NIH})$.

\section{Western Blotting}

Western blotting was performed according to the reference described ${ }^{20}$.

\section{Cell Culture}

Mouse aortic smooth muscle cells and 293T cells were purchased from American Type Culture Collection. Cells were cultured in DMEM supplemented with $10 \%$ serum (Gibco) under a humidified environment at $37^{\circ} \mathrm{C}, 5 \% \mathrm{CO} 2$ and $95 \%$ air. Cells were sub-cultured when grown to $80-90 \%$ confluence. Cells within 10 generations were used for experiments. AMPK-a 1 knock-out KDR/EGFP-Pdlim5 VSMCs was a gift generously provided by Professor Takashima, Osaka University.

\section{Adenoviral infection}

All adenovirus, a replication-defective adenoviral vector expressing wildtype Pdlim5 or Pdlim5 mutant fused with flag or EGFP tag, were gifts from Takashima group, Osaka University. The Pdlim5 S177A or S177D adenoviral vector expressed a mutatant of Pdlim5 in which Serine 177 was substituted with Alanine (S77A) or Aspartate (S77D) respectively. For animal experiments, Diabetic mice were infected with adenovirus in an open-chest myocardium injection after anesthetized by isoflurane (2\%)-oxygen (98\%) mixture. For cellular model work, VSMCs were infected with Ad-Pdlim5 WT, Ad-Pdlim5-S177A, or Ad-Pdlim5-S177D overnight in medium supplemented with $2 \%$ fetal calf serum. The cells were then washed and incubated in fresh VSMCs growth medium without fetal calf serum for an additional $12 \mathrm{~h}$ prior to experimentation. These conditions typically produced an infection efficiency of at least $80 \%$, as determined by EGFP or flag expression.

\section{Scratch assay}


Mouse aortic smooth muscle cells were seeded on $35-\mathrm{mm}$ glass dishes at an initial density of $5 \times 10 \bigotimes$ $\mathrm{cm}^{2}$. A scratch was made with a P-200 pipette tip eight hours after seeding. $1 \mathrm{mM}$ metformin hydrochloride (Sigma-Aldrich) were added after changing the medium, and PBS as a control. The lesions were observed by the Zeiss inverted microscope and measured with the ImageJ software $(\mathrm{NIH})$ once an hour and totally observed for $8 \mathrm{~h}$.

\section{Time-lapse imaging of VSMC cells}

EGFP-Pdlim5-WT cells or EGFP-Pdlim5/AMPK- $\mathbf{\alpha} 1 \mathrm{KO}$ cells were plated on 35-mm glass dishes coated with collagen at an initial density of $4 \times 10^{4} \mathrm{~cm}^{2}$. Five hours after plating, cells were treated with metformin $(1 \mathrm{mM})$. The fluorescence images were recorded as described before ${ }^{20}$.

\section{Statistical analyses}

Data in graphs are presented as means \pm s.e.m. Two-tailed Student's t-test was utilized to compare two groups. Differences among multiple experimental groups were analyzed by one-way analysis of variance, followed by a post-hoc comparison with Dunnett's method utilizing SPSS 16 (IBM). Pख0.05 was considered statistically significant. *Pख0.05, **P囚0.01; ***Pख0.001.

\section{Results}

\section{AMPK-Pdlim5 pathway involves in VSMCs lamellipodia formation in vitro}

To investigate the role of AMPK-Pdlim5 pathway in cell migration, we established the knockdown-rescue (KDR) system in VSMCs as described before ${ }^{20}$, in which endogenous Pdlim5 was replaced with EGFP or flag-fused Pdlim5, Pdlim5 S177A (an unphosphorylatable mutant), or Pdlim5-S177D (a phosphomimetic mutant), respectively. In Fig. 1A, it was shown that metformin could induce phosphorylation of AMPK in vitro. The activation of AMPK was followed by phosphorylation of Pdlim5, whereas Pdlim5-S177A was not phosphorylated (Fig. 1A). Pdlim5-S177D was recognized by the Ab-pS177 antibody even without Met stimulation (Fig. 1A). In Fig. 1B, both KDR/WT- and KDR/ Pdlim5-S177A cells possessed smooth lamellipodia-like edges, whereas KDR/Pdlim5-S177D cells displayed decreased lamellipodia formation and jagged edges with excessive filopodia-like protrusions and ventral stress fibers. In addition, it was found both KDR/WT- and KDR/S177A-Pdlim5 cells had tiny and scattered spots of focal adhesions at the junction between the lamellipodia and lamella; by contrast, in KDR/S177D-Pdlim5 cells, focal adhesions were displaced to the edge of the cell and significantly enlarged in size (Fig. 1C).

\section{AMPK activation induced phosphorylation of Pdlim5 takes part in regulation of VSMCs migration}

To elucidate whether the AMPK-Pdlim5 activation related morphological changes inhibit cell migration, we performed time-lapse imaging with Pdlim5-EGFP overexpressed VSMCs with or without Met stimulation (Fig. 1D). It is shown that metformin reduced lamellipodia formation and promoted enhancement of the EGFP signals from the side opposite the lamellae, a pattern similar to the growth of dorsal stress fibers. This morphological change was similar to that observed in KDR/Pdlim5 S177D cells in Fig. 1b. Next, a scratch assay was performed in the KDR/Pdlim5 cells (Fig. 1E) to observe whether 
decreased lamellipodia and enhanced stress fiber would inhibit cell migration. It was shown that migration ability of KDR/S177D cells is lower compared with KDR/WT- and KDR/ Pdlim5-S177A cells, while Met inhibit both KDR/WT- and KDR/Pdlim5-S177D cells migration but not KDR/S177A cells (Fig. 1F).

To see whether metformin inhibit cell migration through AMPK-Pdlim5 pathway, AMPKo 1 null/WTPdlim5 cells were constructed, and experiments mentioned above were carried out in this cell line. The result in Fig. 2A showed that the absence of AMPK $\mathbf{\alpha} 1$ was followed by reduced phosphorylation of AMPK and Pdlim5 in AMPK $\mathbf{\alpha} 1$ null/WT-Pdlim5 cells. In scratch assay, it is observed that the migratory ability of WT-Pdlim 5 cells is lower than AMPK 1 null/WT-Pdlim 5 cells (Fig. 2B, C). The enhanced stress fiber and reduced VSMCs migration were also observed with time-lapse imaging (Fig. 2D). These findings suggested that Ser177 phosphorylation of Pdlim5 by AMPK induce attenuated lamellipodia formation, enhanced cell adhesion and reduced migration in VSMCs.

\section{Metformin has no influence on VSMC differentiation}

To investigate whether metformin could influence the phenotype switching of VSMCs, wildtype VSMCs were treated with TNF- $\alpha$ and PDGF-BB or metformin combined with those 2 cytokines. It was shown that TNF- $\alpha$ and PDGF-BB increase the expression of phenotype switching regulator KLF4 but reduce the expression of contractile protein SMA and SM22a, while metformin couldn't inhibit this phenomenon (Fig. 2E). This result suggests that activation of AMPK by metformin has no influence on the phenotype switching of VSMCs.

\section{Metformin attenuates intimal hyperplasia after artery injury in Myh11-Cre/Rosa26-EGFP mice}

As metformin could inhibit VSMCs migration in vitro, a wire injury induced-vascular remodeling model was utilized to verify this function of Met in vivo (Fig. 3). To accomplish this experiment, 6- to 8-week-old Myh11-Cre/Rosa26-EGFP mice, which express EGFP in mature VSMCs, were accepted ligation of the left carotid artery. Accumulation of VSMC-derived cells in neointima of left carotid artery was observed by confocal microscopy of arterial cross sections. It was found that many Myh11-expressing VSMCs derived EGFP positive cells contributed to the neo-intima lesions and constituted a significant proportion of the total cell number within lesions. Interestingly, compared with control group, Metformin treatment reduced the intima hyperplasia (Fig. 3A) and patch size (Fig. 3B) compared to saline treatment significantly.

\section{Metformin alleviates Atherosclerotic Lesions in Diabetic ApoE ${ }^{-/-}$Mice}

To study whether metformin could be used to prevent diabetes accelerated atherosclerosis, $\mathrm{ApoE}^{-/-}$mice were induced DM and atherosclerosis with streptozocin and HFD. Diametic mice were treated with metformin was as described in methods (Fig. 4A). It was found that diabetic $\mathrm{ApoE}^{-/-}$mice possessed obviously en face lesions in the aortic arch, thoracic and abdominal aorta and greater atherosclerotic lesions in the aortic root (Fig. 4B-E). Metformin intervention significantly reduced lesion areas in the aortic root and aortic arch in diabetic $\mathrm{ApoE}^{-/-}$mice. SMA positive deposition in aortic root was also smaller in 
metformin treated mice than in diabetic mice, which suggest metformin inhibit VSMCs accumulation in atherosclerotic plaques (Fig. 4F, G).

\section{The activation of AMPK-Pdlim5 pathway is involved in the protective function of metformin against diabetes accelerated atherosclerosis in apo $\mathrm{E}^{-/-}$mice}

To study the role of AMPK-Pdlim5 pathway in the development of atherosclerosis, $\mathrm{ApoE}^{-/-}$animals were divided randomly into streptozocin-induced diabetes mellitus together with or without metformin, Pdlim5 phosphomimetic mutant carried adenovirus (Pdlim5 S177D) or Pdlim5 unphosphotable mutant carried adenovirus (Pdlim5 S177A). It is shown in Fig. 5 that metformin reduced hyperglycemia significantly (Fig. 5A), while exhibit no obviously influence upon dyslipidemia induced by STZ and HFD (Fig. 5B-E). However, the manipulation of Pdlim5 phosphorylation with adenovirus have no significant influence on metabolic disorders in DM mice (Fig. 5A-E). The efficiency of adenovirus upon interference of AMPK activation and phosphorylation of Pdlim5 were approved as in Fig. 5F. Diabetic $\mathrm{ApoE}^{-/-}$mice developed significantly larger en face lesions in the aortic arches (Fig. 5G-J) and larger SMA- and phosphorylated Pdlim5- positive lesions in the aortic roots compared with metformin treatment group (Fig. 6A-C). Pdlim5 negative mutant adenovirus (S177A) alleviate metformin's protective function mentioned above and increase the plaque area significantly (Fig. 5G-J, Fig. 6A-C). However, Pdlim5 constitutively active adenovirus (S177D) suppressed atherosclerosis obviously even under the absence of metformin (Fig. 5GJ, Fig. 6A-C).

\section{Discussion}

Accumulation of vascular smooth muscle cell (VSMC) in the vascular intima is a hallmark of atherosclerosis, but their exact origins are still in controversies ${ }^{8,21}$. In humans, both pre-existing intimal and medial VSMCs can contribute to plaque VSMCs ${ }^{25}$. In mice, the VSMCs in the fibrous cap are unambiguously derived from media, which suggest the importance of cell migration in the pathogenesis of atherosclerosis ${ }^{26}$. This is consistent with our finding that inhibiting VSMCs migration through activation of AMPK reduces neointima formation induced by artery injury and STZ/HFD induced atherosclerosis. The mechanics of VSMC migration in atherosclerotic lesions involves formation of plasma membrane-leading lamellae (leading edge) and disengagement of focal adhesions that are in contact with the $\mathrm{ECM}^{27}$. We found that metformin could inhibit cell migration through enforcement of focal adhesions and reducing lamellipodia formation in vitro. The underlying mechanism has been elucidated by our and others publication that phosphorylation of Pdlim5 by AMPK disrupts the binding between Pdlim5 with Arhgef 6 at the cell periphery ${ }^{20}$. The dissociation suppresses Rac1 activity and dislocates the Arp2/3 complex from the leading edge of cell which impairs the lamellipodia formation and cell migration $20,27,28$.

VSMC migration in atherosclerosis has also been related to phenotype-switching, a synthetic, dedifferentiated state ${ }^{29}$. The phenotype switched VSMCs exhibits reduced production of contractile proteins 
but higher expression of ECM related products, increased levels of secretory organelles and proinflammatory cytokines ${ }^{30}$. At the molecular level, the VSMC phenotype-transition is governed by transcription factors myocardin-serum response factor (SRF) 58 and Krüppel-like factor 4 (KLF4) 22, 31 . Diabetes mellitus (DM) associated pathological factors exacerbate the synthetic phenotype of VSMCs through up-regulation of KLF4 ${ }^{32}$. Although our work shown that metformin induced AMPK activation has no influence on expression of KLF4 and 'contractile proteins' (Fig. 2E), the relationship between VSMCs migration and phenotype-switching in atherosclerotic plaques still needs further investigation.

AMP-activated protein kinase (AMPK) is a vital enzyme for regulating cellular energy homeostasis ${ }^{33}$. Activation of AMPK depends on phosphorylation at its T172 site and binding with AMP and/or ADP 34 . Compelling evidence has indicated an inverse correlation between diabetes and AMPK activity ${ }^{33}$. Therefore, AMPK-activating agents have the potential to be utilized as precaution or therapies against diabetes and DM related complications. Indeed, metformin, an indirect AMPK activator in addition to T2DM drug, could reduce atherosclerosis in patients with diabetes ${ }^{18,34}$. However, the underlying mechanism is not clear yet. Recent work found that AMPK also plays an important role in the regulation of cell polarity and motility ${ }^{20}$, which throws a light on the research of metformin's anti-atherosclerosis function. In this study, we found that metformin activates AMPK, which phosphorylates Pdlim5 at Ser177, resulting in attenuation of lamellipodia formation and inhibition of vascular smooth muscle cell migration from the medial to intima. We also demonstrated that metformin reduces VSMCs accumulation in atherosclerotic plaques via an AMPK-Pdlim5 dependent manner in STZ and HFDinduced diabetic $\mathrm{ApoE}^{-/-}$mice. It is consistent with existed concept that metformin has multiple beneficial effects on vascular cells (endothelial cells, vascular smooth muscle cells and macrophages), many of which are AMPK-mediated $14,35,36,37,38$. As metformin has been proven safe for decades in clinical practice, it has the potential to be used as a precaution against atherosclerosis in addition to glucose reduction. However, there are still lots of work to be accomplished before translating the knowledge of atherogenesis derived from animal models to human.

\section{Conculsion}

Our research revealed that augmentation of AMPK activity could inhibits VSMCs migration from media to atherosclerotic plaques through phosphorylation of Pdlim5. It may be useful to develop novel therapies towards atherosclerosis and other complications in diabetes.

\section{Abbreviations}

AMPK: AMP activated protein kinase; CVD: Cardiovascular disease; DM: Diabetic mellitus; ECM: Extracellular matrix; KDR: Knockdown-rescue; LDL: Low density lipoprotein; STZ: Streptozotocin; Met: Metformin; Pdlim5: PDZ and LIM domain 5; T2DM: Type 2 diabetic mellitus; VSMCs: Vascular smooth muscle cells. 


\section{Declarations}

\section{Acknowledgement}

We thank Professor Seiji Takashima and Professor. Osamu Tsukamoto, Department of Medical Biochemistry, Osaka University Graduate School of Medicine, Japan, for the advice on whole study design and the gifts of antibodies and plasmids.

We thank Professor Yulin Liao and Professor Jianping Bin, Department of Cardiology, Southern Hospital, Southern Medical University, China, for the generous share of lab equipment.

\section{Authors' Contributions}

Yi Yan, Wei Wu, Zhiyong Zhang conceived and designed the studies, wrote the manuscript and completed all analyses. Yi Yan and Ting Li contribute equally and performed the experiments and data analysis. Zhonghao Li, Mingyuan He, Dejiang Wang, Yingyi Xu, Xuewen Yang, Yuanyuan Bai, Yi Lao contributed to the acquisition of data. All authors approve the final version of the submitted manuscript.

\section{Funding}

This work was supported by the National Natural Science Foundation of China [81870320 and 81600349], Grants of State Key Laboratory of Organ Failure Research [G818NF0029], Guangzhou Science and Technology Innovation Committee Fund [201804010008] and Featured Clinical Technique of Guangzhou [20190337].

\section{Availability of data and materials}

The datasets used and/or analyzed during the current study are available from the corresponding author upon reasonable request.

\section{Ethics approval and consent to participate}

All animal experiments were approved and performed according to Institutional Animal Care and Use Committee (IACUC) of Southern Medical University, which conform to the guidelines from Directive 2010/63/EU of the European Parliament on the protection of animals used for scientific purposes.

\section{Consent for publication}

Not applicable.

\section{Competing interests}

None declared.

\section{Author details}


a. Department of Cardiology, Translational Research Centre of Regenerative Medicine and 3D Printing of Guangzhou Medical University, The Third Affiliated Hospital of Guangzhou Medical University, Guangzhou, 510150, PR China; b. State Key Laboratory of Organ Failure Research, Southern Medical University, Guangzhou, 510000, PR China; c. Department of Pathophysiology, Key Lab for Shock and Microcirculation Research of Guangdong, Southern Medical University. Guangzhou, 510515, PR China; d. Guangzhou First People's Hospital, Guangzhou, 510000, PR China; e. Department of Cardiology, Zhong Shan Hospital of Sun Yat-sen University, Zhongshan City, 528403, PR China.

\section{References}

1. Virmani R, Burke AP, Kolodgie F. Morphological characteristics of coronary atherosclerosis in diabetes mellitus. Can. J. Cardiol. 2006.

2. Chait A, Bornfeldt KE. Diabetes and atherosclerosis: Is there a role for hyperglycemia? J. Lipid Res. 2009.

3. Katakami N. Mechanism of development of atherosclerosis and cardiovascular disease in diabetes mellitus. J. Atheroscler. Thromb. 2018.

4. Gimbrone MA, García-Cardeña G. Endothelial Cell Dysfunction and the Pathobiology of Atherosclerosis. Circ Res 2016;118:620-636.

5. Libby P, Ridker PM, Hansson GK. Progress and challenges in translating the biology of atherosclerosis. Nature. 2011.

6. Libby P. Inflammation in atherosclerosis. Arterioscler Thromb Vasc Bio/ 2012;

7. Basatemur GL, Jørgensen HF, Clarke MCH, Bennett MR, Mallat Z. Vascular smooth muscle cells in atherosclerosis. Nat. Rev. Cardiol. 2019.

8. Bennett MR, Sinha S, Owens GK. Vascular Smooth Muscle Cells in Atherosclerosis. Circ Res 2016;

9. Lusis AJ. Atherosclerosis. Nature. 2000.

10. Bornfeldt KE, Tabas I. Insulin resistance, hyperglycemia, and atherosclerosis. Cell Metab. 2011.

11. Gæde P, Lund-Andersen H, Parving HH, Pedersen O. Effect of a multifactorial intervention on mortality in type 2 diabetes. N Engl J Med 2008;

12. Holman RR, Paul SK, Bethel MA, Matthews DR, Neil HAW. 10-Year follow-up of intensive glucose control in type 2 diabetes. N Engl J Med 2008;

13. Hong J, Zhang Y, Lai S, Lv A, Su Q, Dong Y, Zhou Z, Tang W, Zhao J, Cui L, Zou D, Wang D, Li H, Liu C, Wu G, Shen J, Zhu D, Wang W, Shen W, Ning G. Effects of metformin versus glipizide on cardiovascular outcomes in patients with type 2 diabetes and coronary artery disease. Diabetes Care 2013;

14. Wang Q, Zhang M, Torres G, Wu S, Ouyang C, Xie Z, Zou MH. Metformin suppresses diabetesaccelerated atherosclerosis via the inhibition of Drp1-mediated mitochondrial fission. Diabetes 2017;

15. Duckworth W, Abraira C, Moritz T, Reda D, Emanuele N, Reaven PD, Zieve FJ, Marks J, Davis SN, Hayward R, Warren SR, Goldman S, McCarren M, Vitek ME, Henderson WG, Huang GD. Glucose 
control and vascular complications in veterans with type 2 diabetes. N Engl J Med 2009;

16. Patel A, MacMahon S, Chalmers J, Neal B, Billot L, Woodward M, Marre M, Cooper M, Glasziou P, Grobbee D, Hamet P, Harrap S, Heller S, Liu L, Mancia G, Mogensen CE, Pan C, Poulter N, Rodgers A, Williams B, Bompoint S, Galan BE De, Joshi R, Travert F. Intensive blood glucose control and vascular outcomes in patients with type 2 diabetes. N Engl J Med 2008;

17. Gerstein HC, Miller ME, Byington RP, Goff DC, Bigger JT, Buse JB, Cushman WC, Genuth S, IsmailBeigi F, Grimm RH, Probstfield JL, Simons-Morton DG, Friedewald WT, Gotto AM, Bailey K, Gohdes D, Haffner S, Hiss R, Jamerson K, Lee K, Nathan D, Sowers J, Walters L, Friedewald WT, Buse JB, Bigger JT, Byington RP, Cushman WC, Gerstein HC, Ginsberg HN, et al. Effects of intensive glucose lowering in type 2 diabetes. N Engl J Med 2008;

18. Saisho Y. Metformin and Inflammation: Its Potential Beyond Glucose-lowering Effect. Endocrine, Metab Immune Disord Targets 2015;

19. Potente M, Gerhardt H, Carmeliet P. Basic and therapeutic aspects of angiogenesis. Cell. 2011.

20. Yan Y, Tsukamoto O, Nakano A, Kato H, Kioka H, Ito N, Higo S, Yamazaki S, Shintani Y, Matsuoka K, Liao Y, Asanuma H, Asakura M, Takafuji K, Minamino T, Asano Y, Kitakaze M, Takashima S. Augmented AMPK activity inhibits cell migration by phosphorylating the novel substrate Pdlim5. Nat Commun 2015;

21. Chappell J, Harman JL, Narasimhan VM, Yu H, Foote K, Simons BD, Bennett MR, Jørgensen HF. Extensive Proliferation of a Subset of Differentiated, yet Plastic, Medial Vascular Smooth Muscle Cells Contributes to Neointimal Formation in Mouse Injury and Atherosclerosis Models. Circ Res 2016;

22. Shankman LS, Gomez D, Cherepanova OA, Salmon M, Alencar GF, Haskins RM, Swiatlowska P, Newman AAC, Greene ES, Straub AC, Isakson B, Randolph GJ, Owens GK. KLF4-dependent phenotypic modulation of smooth muscle cells has a key role in atherosclerotic plaque pathogenesis. Nat Med 2015;

23. Lindner V, Fingerle J, Reidy MA. Mouse model of arterial injury. Circ Res 1993;

24. Yang F, Chen Q, Yang M, Maguire EM, Yu X, He S, Xiao R, Wang CS, An W, Wu W, Zhou Y, Xiao Q, Zhang L. Macrophage-derived MMP-8 determines smooth muscle cell differentiation from adventitia stem/progenitor cells and promotes neointima hyperplasia. Cardiovasc Res 2020;

25. Chung IM, Schwartz SM, Murry CE. Clonal architecture of normal and atherosclerotic aorta: Implications for atherogenesis and vascular development. Am J Pathol 1998;

26. Jacobsen K, Lund MB, Shim J, Gunnersen S, Füchtbauer E-M, Kjolby M, Carramolino L, Bentzon JF. Diverse cellular architecture of atherosclerotic plaque derives from clonal expansion of a few medial SMCs. JCl Insight 2017;

27. Gerthoffer WT. Mechanisms of vascular smooth muscle cell migration. Circ. Res. 2007.

28. Louis SF, Zahradka P. Vascular smooth muscle cell motility: From migration to invasion. Exp. Clin. Cardiol. 2010. 
29. Poole JC, Cromwell SB, Benditt EP. Behavior of smooth muscle cells and formation of extracellular structures in the reaction of arterial walls to injury. Am J Pathol 1971;

30. Clarke MCH, Littlewood TD, Figg N, Maguire JJ, Davenport AP, Goddard M, Bennett MR. Chronic apoptosis of vascular smooth muscle cells accelerates atherosclerosis and promotes calcification and medial degeneration. Circ Res 2008;

31. Pipes GCT, Creemers EE, Olson EN. The myocardin family of transcriptional coactivators: Versatile regulators of cell growth, migration, and myogenesis. Genes Dev. 2006.

32. Xi G, Wai C, White MF, Clemmons DR. Down-regulation of insulin receptor substrate 1 during hyperglycemia induces vascular smooth muscle cell dedifferentiation. J Biol Chem 2017;

33. Jeon SM. Regulation and function of AMPK in physiology and diseases. Exp. Mol. Med. 2016.

34. Grahame HD. AMPK: A target for drugs and natural products with effects on both diabetes and cancer. Diabetes. 2013.

35. Seneviratne A, Cave L, Hyde G, Moestrup SK, Carling D, Mason JC, Haskard DO, Boyle JJ. Metformin directly suppresses atherosclerosis in normoglycemic mice via haematopoietic Adenosine Monophosphate-Activated Protein Kinase (AMPK). Cardiovasc Res 2020;

36. Wan X, Huo Y, Johns M, Piper E, Mason JC, Carling D, Haskard DO, Boyle JJ. 5'-AMP-activated protein kinase-activating transcription factor 1 cascade modulates human monocyte-derived macrophages to atheroprotective functions in response to heme or metformin. Arterioscler Thromb Vasc Biol 2013;

37. Messaoudi S El, Rongen GA, Boer RA De, Riksen NP. The cardioprotective effects of metformin. Curr. Opin. Lipidol. 2011.

38. Foretz M, Guigas B, Bertrand L, Pollak M, Viollet B. Metformin: From mechanisms of action to therapies. Cell Metab. 2014.

\section{Figures}


A

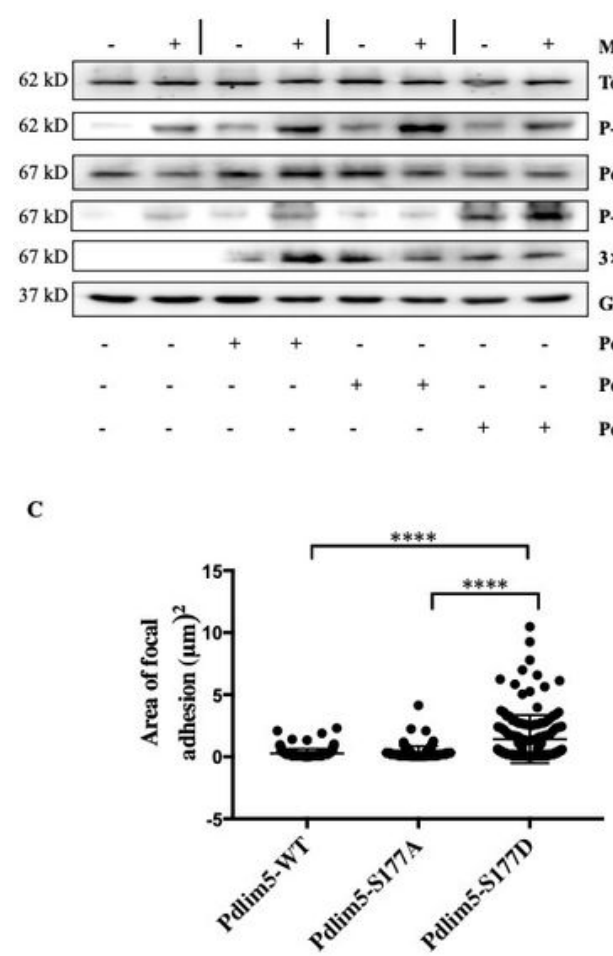

B

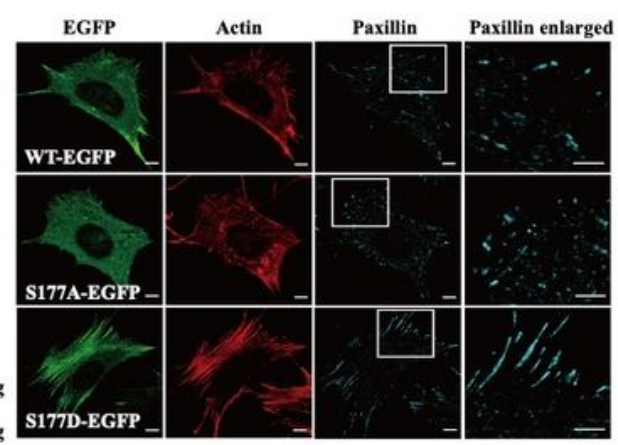

D

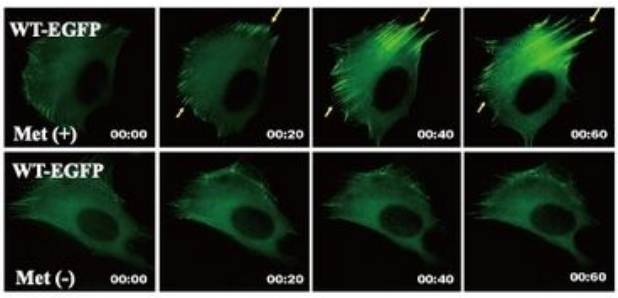

E

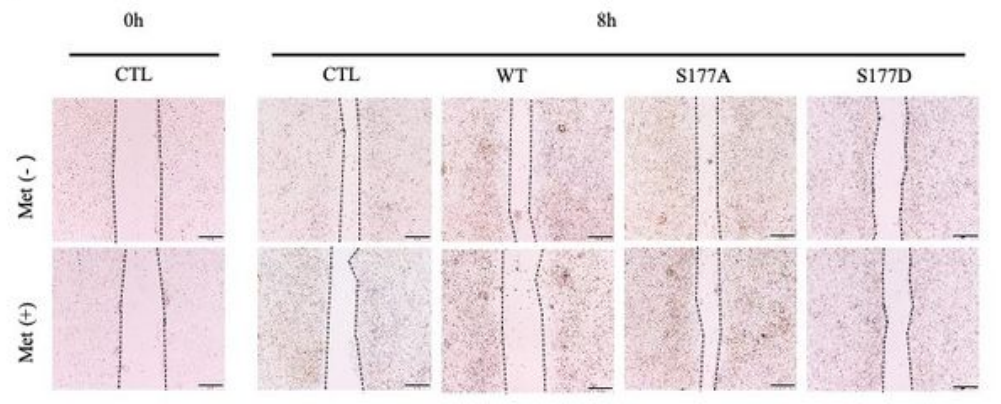

F

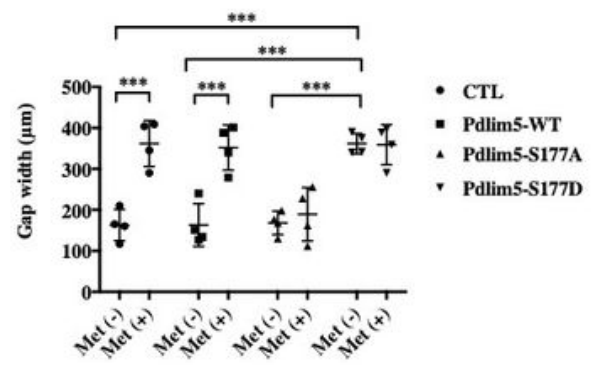

Figure 1

Activation of AMPK-Pdlim5 pathway inhibits VSMCs migration. (A) Metformin Activate of AMPK-Pdlim5 signaling pathway. VSMCs were transfected with CTL siRNA or Pdlim5 siRNA. Pdlim5 siRNA-resistant flag-Pdlim5 (WT, S177A or S177D) was added back by adenoviral-mediated gene delivery. Phosphorylation level of AMPK and Pdlim5 were detected with western blotting. (B) GFP signal and immunostaining of actin and paxillin in KDR/EGFP-Pdlim5 cells (WT, S177A and S177D). Actin and 
paxillin were stained with phalloidin and anti-paxillin antibody, to display actin microfilaments and focal adhesions, respectively. Th right panels are the magnified images of the region outlined by white boxes. Scale bar, $10 \square \mathrm{m}$. (C) Focal adhesion area of KDR/EGFP-Pdlim5 cells (WT, S177A and S177D) were measured according to paxillin-positive region in fig 2B and compared using one-way analysis of variance, followed by a post-hoc comparison with Dunnett's method for multiple comparisons. $P^{*}<0.05$, $P * \star<0.01, n=100$. (D) Time-lapse images of GFP signal in KDR/EGFP-WT-Pdlim5 cells with or without metformin stimulation $(1 \mathrm{mM})$. Yellow arrowheads indicate growing of dorsal stress fibers from the opposite side in KDR/EGFP-WT-Pdlim5 cells after metformin stimulation. Scale bar, $10 \square \mathrm{m}$. (E) Scratch assay of metformin stimulated KDR/EGFP-Pdlim5 cells. Phase-contrast images of KDR/Pdlim5 cells (WT, S177A and S177D) $8 \mathrm{~h}$ after scratching with or without metformin. Scale bar, $0.5 \mathrm{~mm}$. (F) Bar graph showing the gap width $8 \mathrm{~h}$ after scratching (from $\mathrm{E}$ ). Data are representative of means \pm s.e.m. from four independent experiments. Significance of results was assessed using one-way analysis of variance, followed by a post-hoc comparison with Dunnett's method for multiple comparisons. $P^{\star \star \star}<0.001, n=4$. 
$\mathbf{A}$

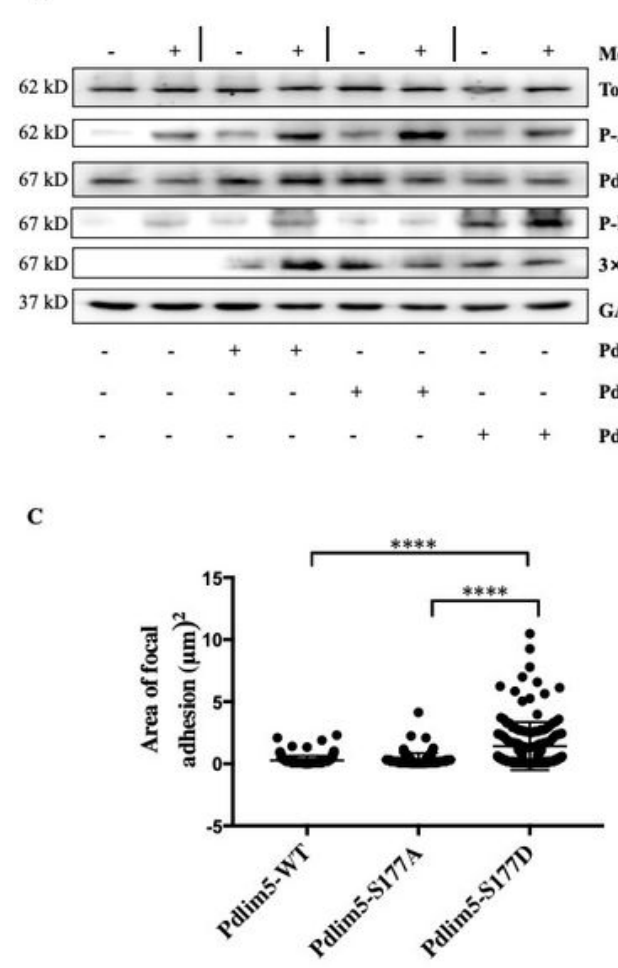

B

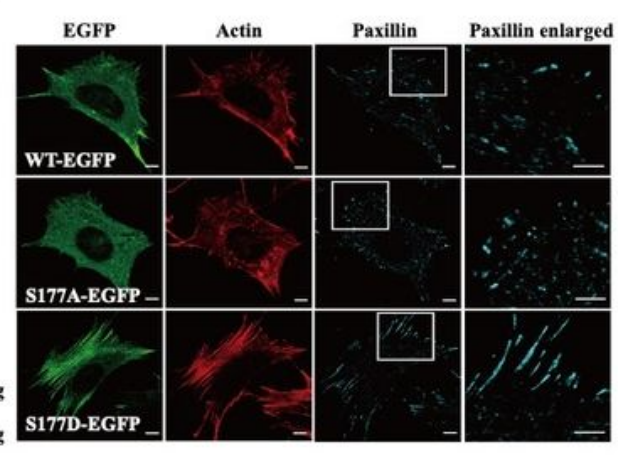

D

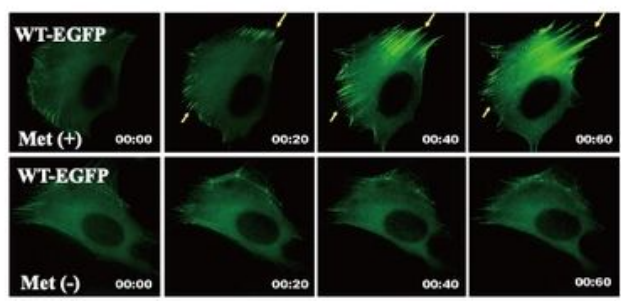

E

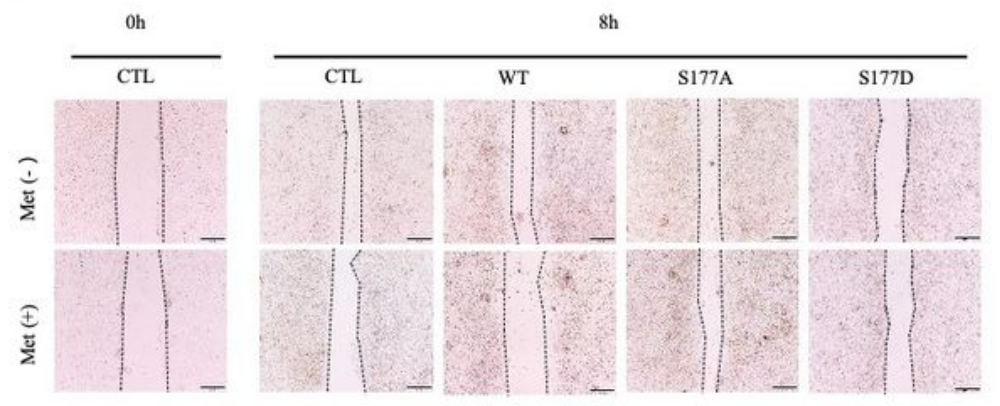

F

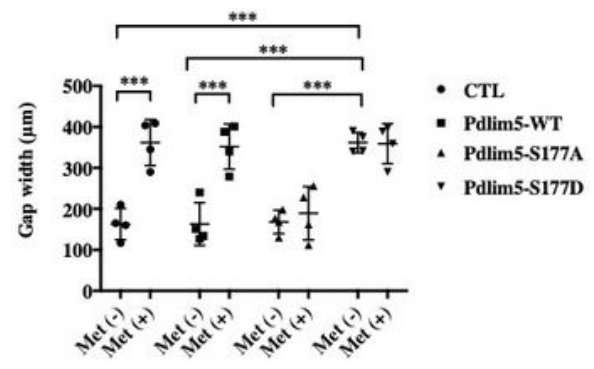

Figure 1

Activation of AMPK-Pdlim5 pathway inhibits VSMCs migration. (A) Metformin Activate of AMPK-Pdlim5 signaling pathway. VSMCs were transfected with CTL siRNA or Pdlim5 siRNA. Pdlim5 siRNA-resistant flag-Pdlim5 (WT, S177A or S177D) was added back by adenoviral-mediated gene delivery. Phosphorylation level of AMPK and Pdlim5 were detected with western blotting. (B) GFP signal and immunostaining of actin and paxillin in KDR/EGFP-Pdlim5 cells (WT, S177A and S177D). Actin and 
paxillin were stained with phalloidin and anti-paxillin antibody, to display actin microfilaments and focal adhesions, respectively. Th right panels are the magnified images of the region outlined by white boxes. Scale bar, $10 \square \mathrm{m}$. (C) Focal adhesion area of KDR/EGFP-Pdlim5 cells (WT, S177A and S177D) were measured according to paxillin-positive region in fig 2B and compared using one-way analysis of variance, followed by a post-hoc comparison with Dunnett's method for multiple comparisons. $P^{*}<0.05$, $P * \star<0.01, n=100$. (D) Time-lapse images of GFP signal in KDR/EGFP-WT-Pdlim5 cells with or without metformin stimulation $(1 \mathrm{mM})$. Yellow arrowheads indicate growing of dorsal stress fibers from the opposite side in KDR/EGFP-WT-Pdlim5 cells after metformin stimulation. Scale bar, $10 \square \mathrm{m}$. (E) Scratch assay of metformin stimulated KDR/EGFP-Pdlim5 cells. Phase-contrast images of KDR/Pdlim5 cells (WT, S177A and S177D) $8 \mathrm{~h}$ after scratching with or without metformin. Scale bar, $0.5 \mathrm{~mm}$. (F) Bar graph showing the gap width $8 \mathrm{~h}$ after scratching (from $\mathrm{E}$ ). Data are representative of means \pm s.e.m. from four independent experiments. Significance of results was assessed using one-way analysis of variance, followed by a post-hoc comparison with Dunnett's method for multiple comparisons. $P^{\star \star \star}<0.001, n=4$. 
A

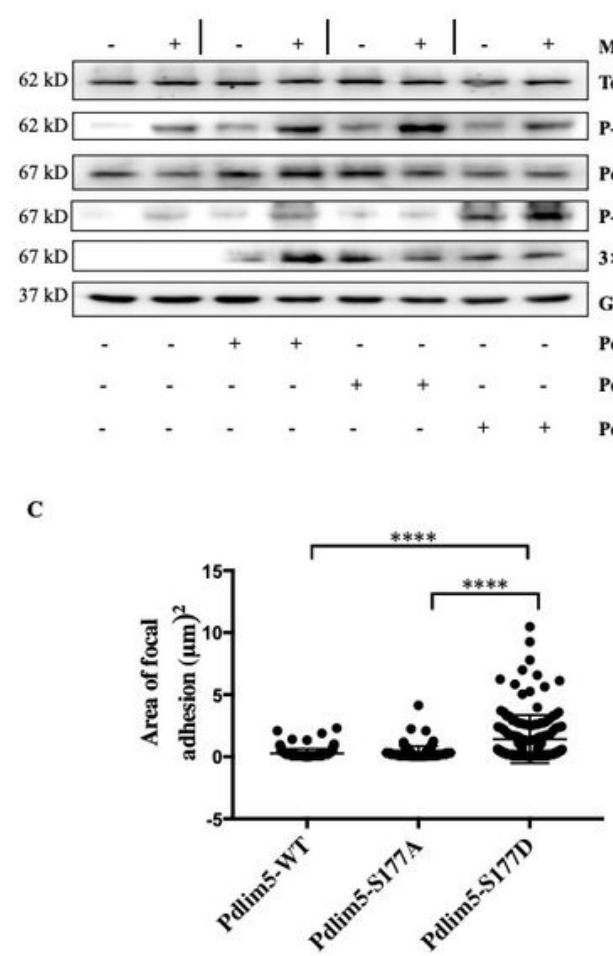

B

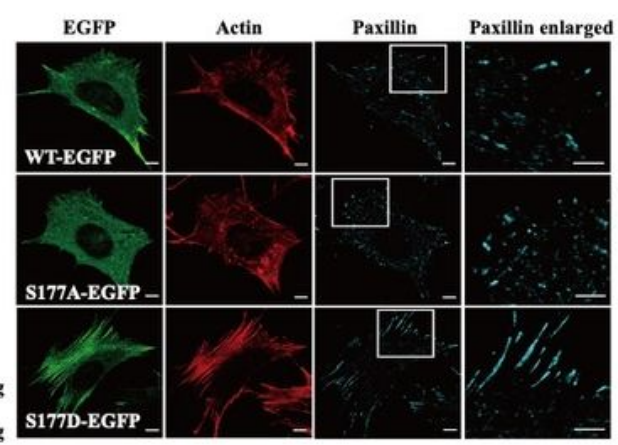

D

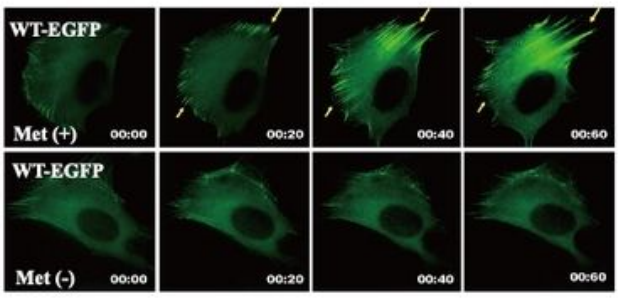

E

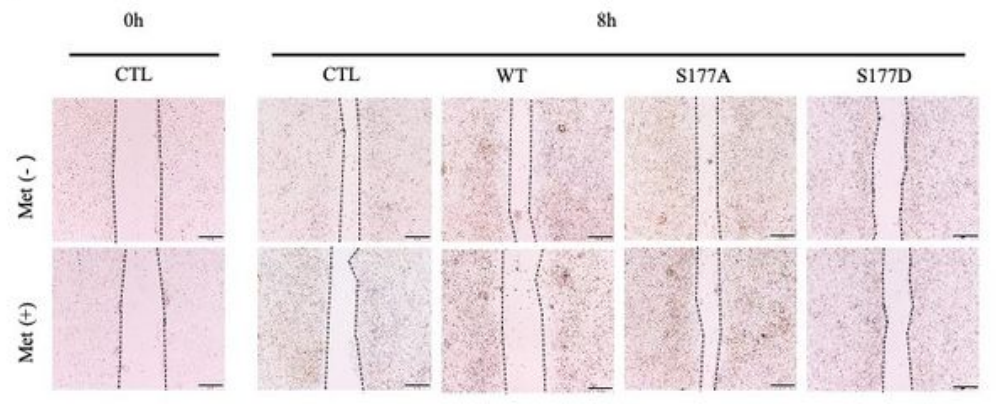

F

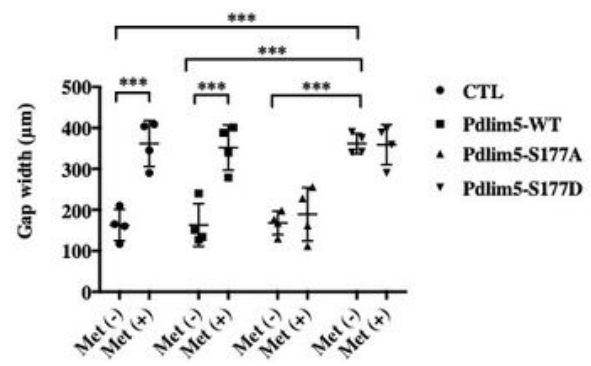

Figure 1

Activation of AMPK-Pdlim5 pathway inhibits VSMCs migration. (A) Metformin Activate of AMPK-Pdlim5 signaling pathway. VSMCs were transfected with CTL siRNA or Pdlim5 siRNA. Pdlim5 siRNA-resistant flag-Pdlim5 (WT, S177A or S177D) was added back by adenoviral-mediated gene delivery. Phosphorylation level of AMPK and Pdlim5 were detected with western blotting. (B) GFP signal and immunostaining of actin and paxillin in KDR/EGFP-Pdlim5 cells (WT, S177A and S177D). Actin and 
paxillin were stained with phalloidin and anti-paxillin antibody, to display actin microfilaments and focal adhesions, respectively. Th right panels are the magnified images of the region outlined by white boxes. Scale bar, $10 \square \mathrm{m}$. (C) Focal adhesion area of KDR/EGFP-Pdlim5 cells (WT, S177A and S177D) were measured according to paxillin-positive region in fig 2B and compared using one-way analysis of variance, followed by a post-hoc comparison with Dunnett's method for multiple comparisons. $P^{*}<0.05$, $P * \star<0.01, n=100$. (D) Time-lapse images of GFP signal in KDR/EGFP-WT-Pdlim5 cells with or without metformin stimulation $(1 \mathrm{mM})$. Yellow arrowheads indicate growing of dorsal stress fibers from the opposite side in KDR/EGFP-WT-Pdlim5 cells after metformin stimulation. Scale bar, $10 \square \mathrm{m}$. (E) Scratch assay of metformin stimulated KDR/EGFP-Pdlim5 cells. Phase-contrast images of KDR/Pdlim5 cells (WT, S177A and S177D) $8 \mathrm{~h}$ after scratching with or without metformin. Scale bar, $0.5 \mathrm{~mm}$. (F) Bar graph showing the gap width $8 \mathrm{~h}$ after scratching (from $\mathrm{E}$ ). Data are representative of means \pm s.e.m. from four independent experiments. Significance of results was assessed using one-way analysis of variance, followed by a post-hoc comparison with Dunnett's method for multiple comparisons. $P^{\star \star \star}<0.001, n=4$. 
A

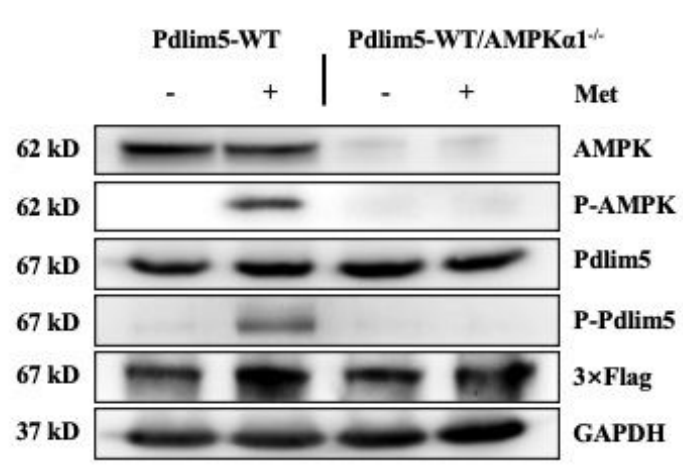

C

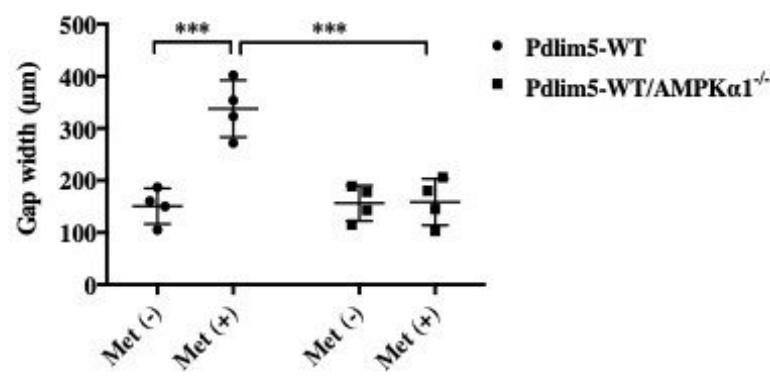

$\mathbf{E}$

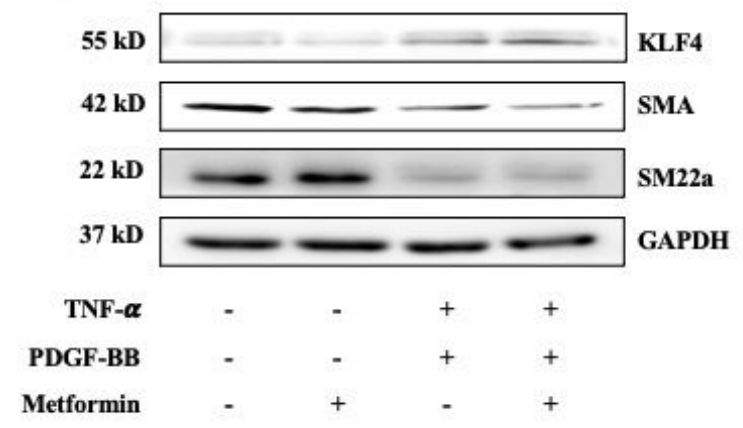

B

0h

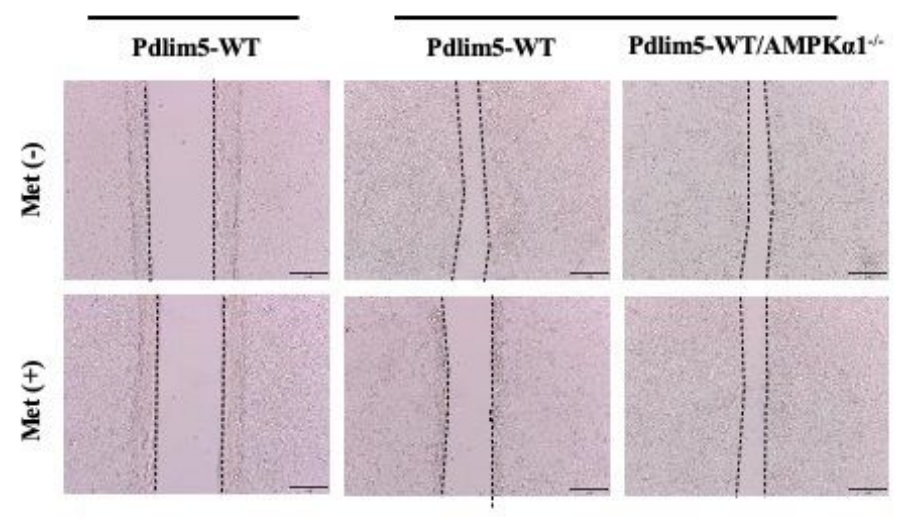

D

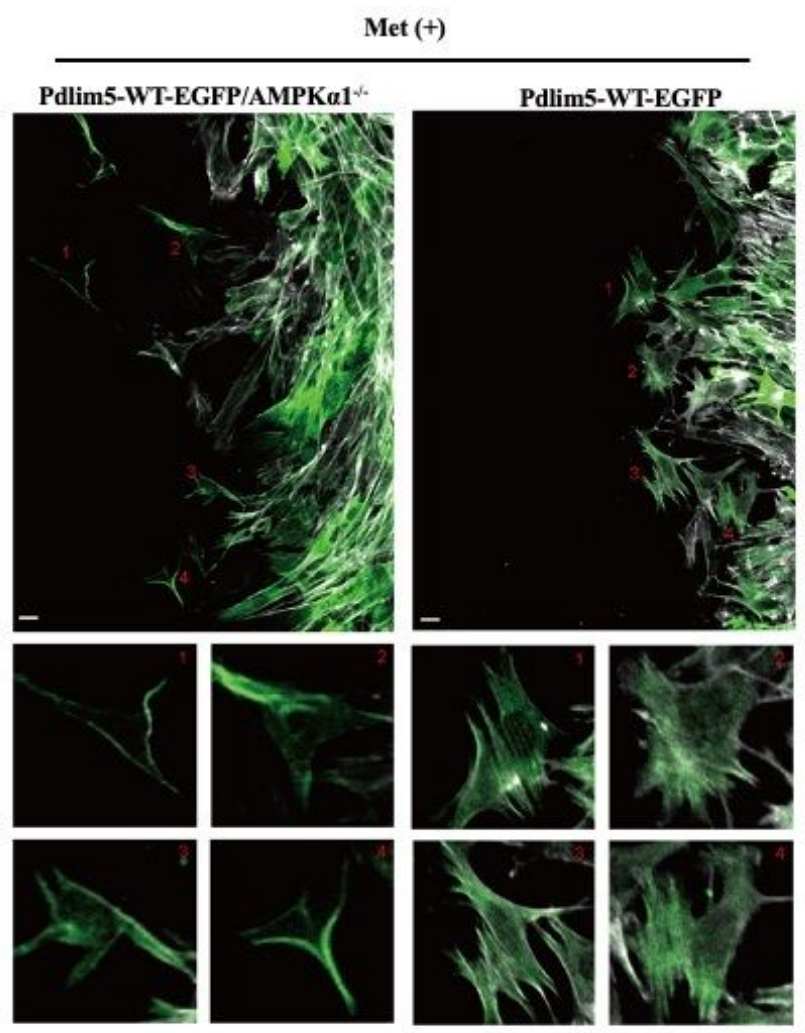

\section{Figure 2}

Metformin inhibits VSMCs migration through AMPK. (A) KDR/Flag-WT-Pdlim5 VSMCs and AMPK $\alpha 1$ null/KDR/EGFP-WT-Pdlim5 VSMCs were stimulated with metformin before detecting phosphorylation level of AMPK and Pdlim5 with immunoblotting. (B) KDR/EGFP-Pdlim5 VSMCs and AMPK $\alpha 1$ null/KDR/EGFP -Pdlim5 VSMCs were stimulated with metformin ( $1 \mathrm{mM})$ for 8 hours in a scratch assay. (C) Bar graph showing the gap width $8 \mathrm{~h}$ after scratching (from B). Data are representative of 
means \pm s.e.m. from four independent experiments. Significance of differences between series of results was assessed using one-way analysis of variance, followed by a post-hoc comparison with Dunnett's method for multiple comparisons. $P^{\star * *}<0.001, n=4$. (D) GFP images of AMPK $\alpha 1$-null/KDR/EGFP-WTPdlim5 cells before and after metformin stimulation ( $1 \mathrm{mM})$. Magnified images below show the cells labelled by the number. Scale bar, $10 \square \mathrm{m}$. (E) Western blot of VSMC contractile markers and KLF4 in control, TNF- $\alpha$ and PDGF-BB treated, and metformin combined with those 2 cytokines treated VSMCs.

A

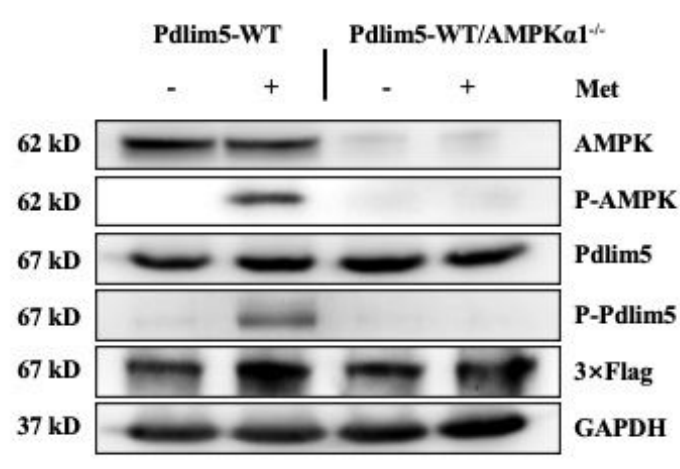

C

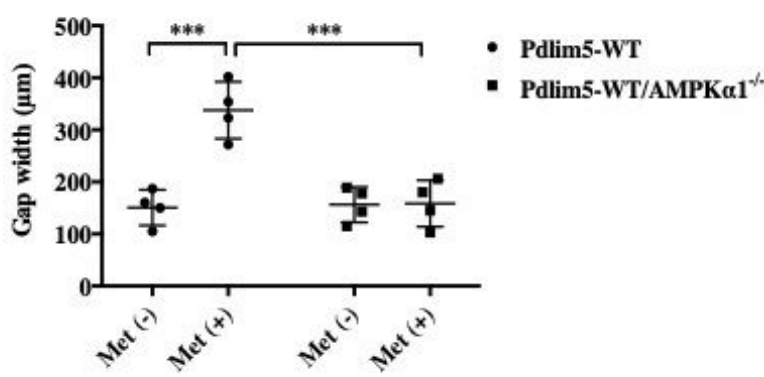

$\mathbf{E}$

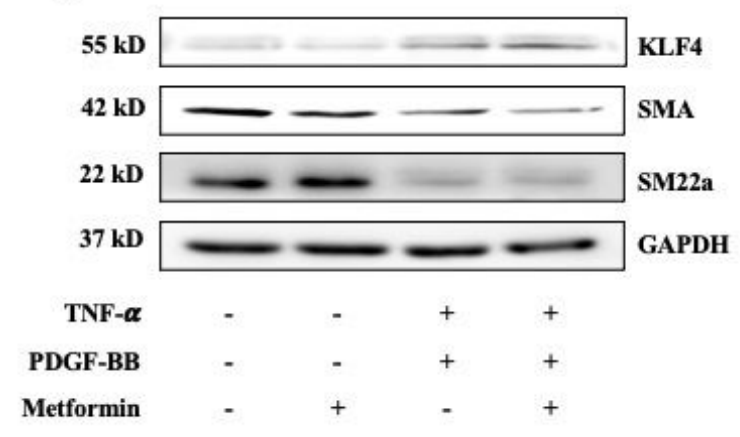

B

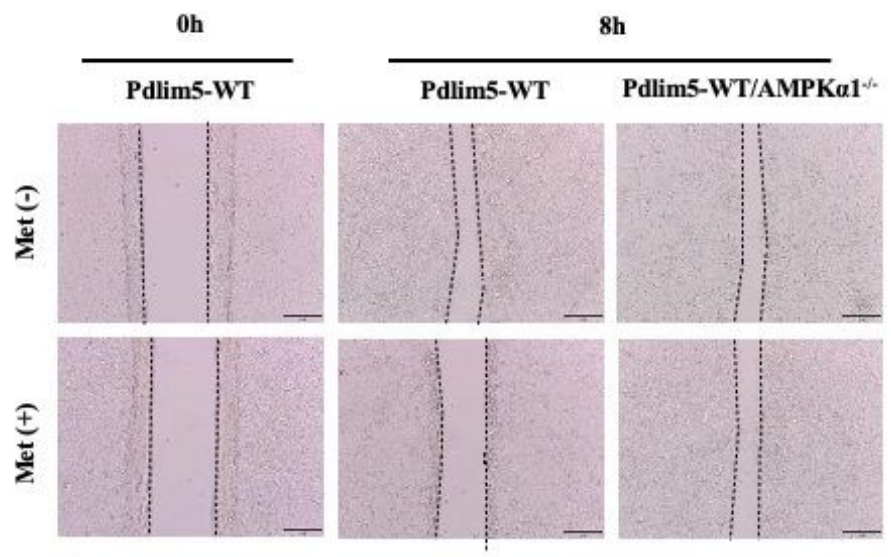

D

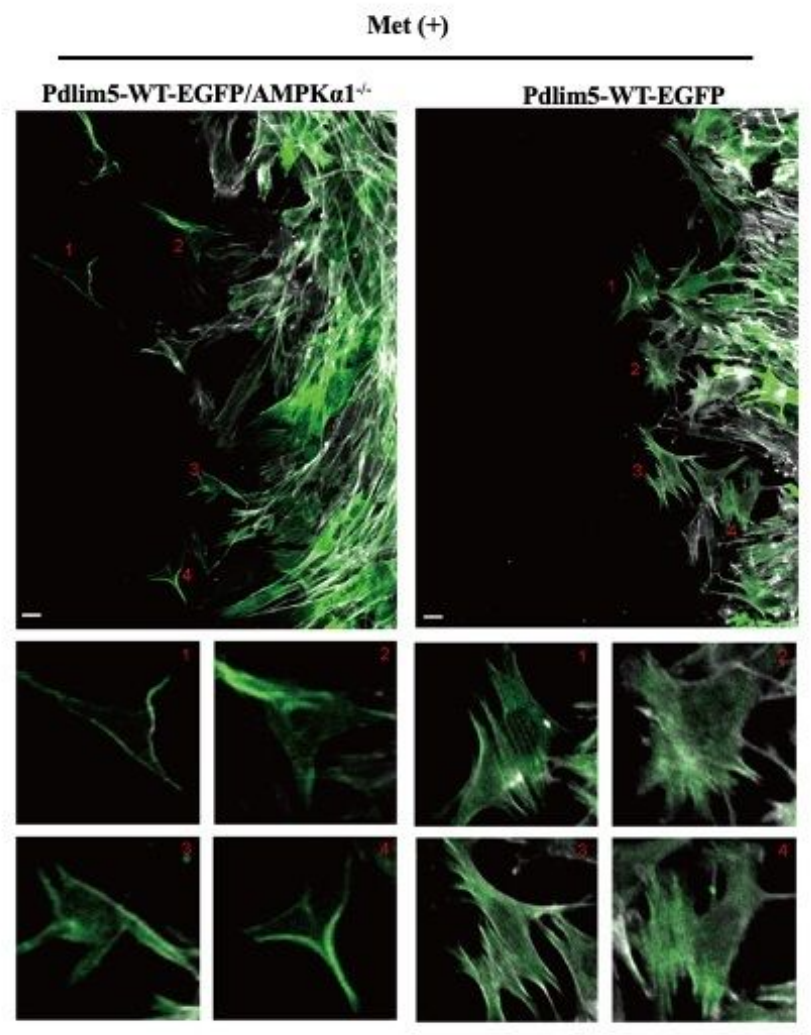


Metformin inhibits VSMCs migration through AMPK. (A) KDR/Flag-WT-Pdlim5 VSMCs and AMPK $\alpha 1$ null/KDR/EGFP-WT-Pdlim5 VSMCs were stimulated with metformin before detecting phosphorylation level of AMPK and Pdlim5 with immunoblotting. (B) KDR/EGFP-Pdlim5 VSMCs and AMPK $\alpha 1-$ null/KDR/EGFP -Pdlim5 VSMCs were stimulated with metformin ( $1 \mathrm{mM})$ for 8 hours in a scratch assay. (C) Bar graph showing the gap width $8 \mathrm{~h}$ after scratching (from B). Data are representative of means \pm s.e.m. from four independent experiments. Significance of differences between series of results was assessed using one-way analysis of variance, followed by a post-hoc comparison with Dunnett's method for multiple comparisons. $P^{\star * *}<0.001, n=4$. (D) GFP images of AMPK $\alpha 1$-null/KDR/EGFP-WTPdlim5 cells before and after metformin stimulation $(1 \mathrm{mM})$. Magnified images below show the cells labelled by the number. Scale bar, $10 \mathrm{Im}$. (E) Western blot of VSMC contractile markers and KLF4 in control, TNF- $\boldsymbol{\alpha}$ and PDGF-BB treated, and metformin combined with those 2 cytokines treated VSMCs. 
A

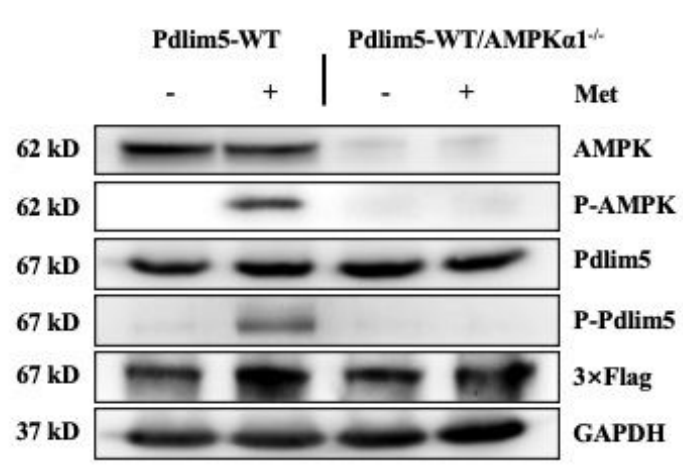

C

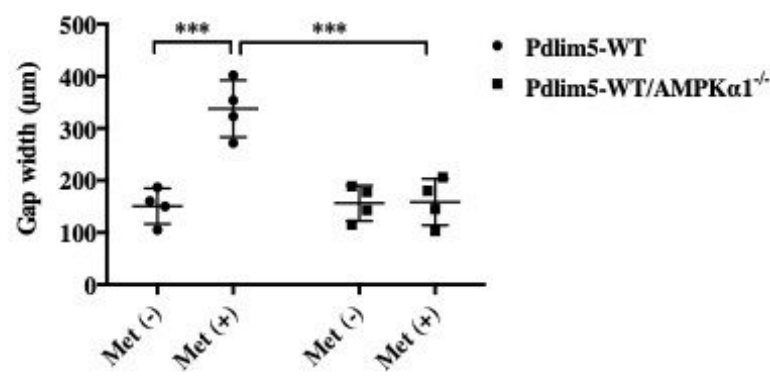

$\mathbf{E}$

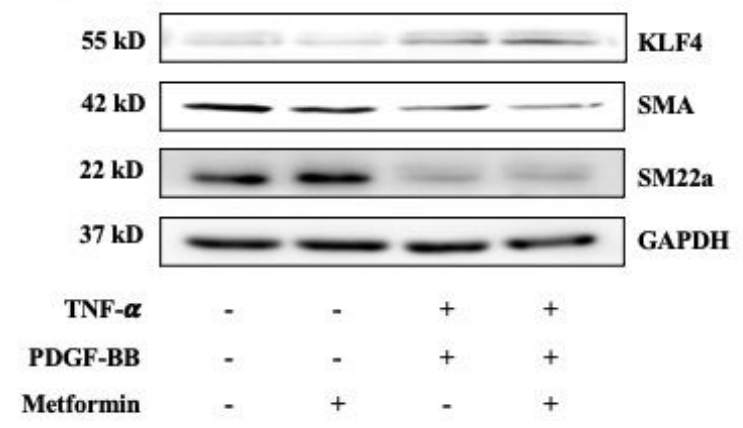

B oh

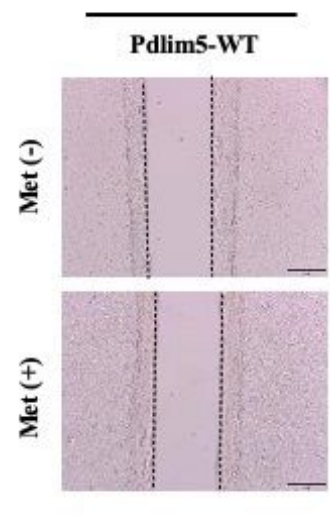

$8 \mathrm{~h}$

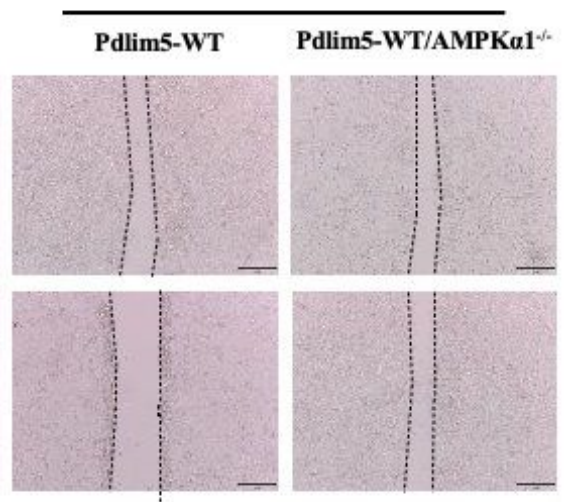

D
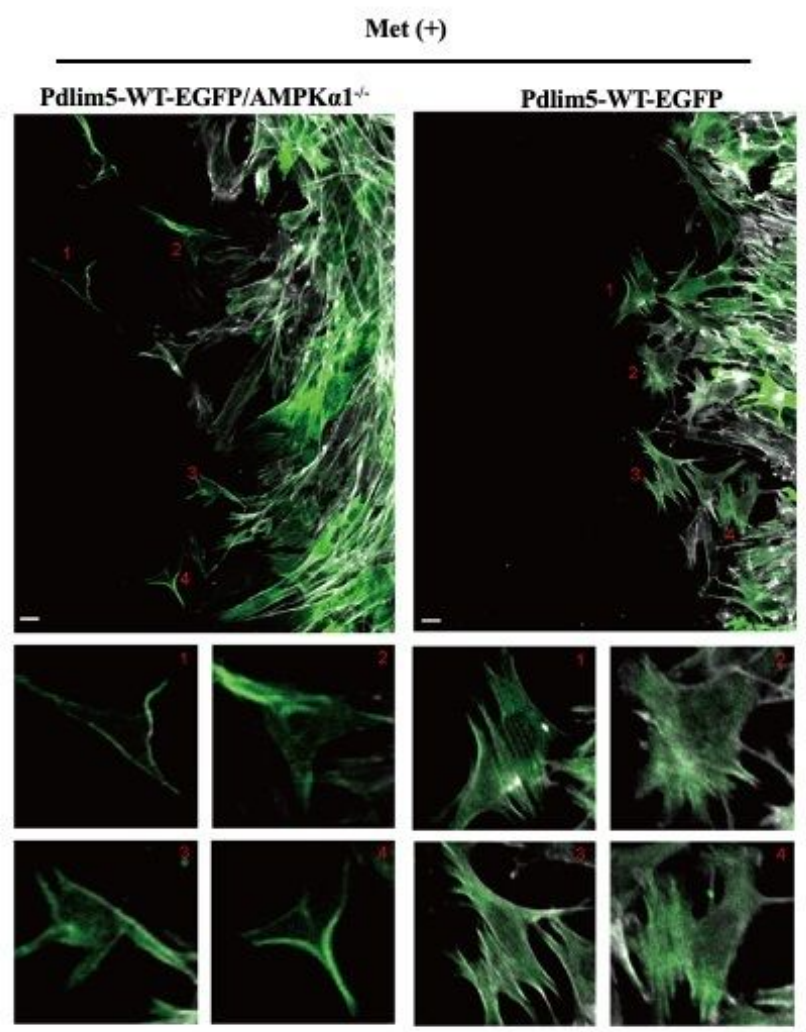

\section{Figure 2}

Metformin inhibits VSMCs migration through AMPK. (A) KDR/Flag-WT-Pdlim5 VSMCs and AMPK $\alpha 1$ null/KDR/EGFP-WT-Pdlim5 VSMCs were stimulated with metformin before detecting phosphorylation level of AMPK and Pdlim5 with immunoblotting. (B) KDR/EGFP-Pdlim5 VSMCs and AMPK $\alpha 1$ null/KDR/EGFP -Pdlim5 VSMCs were stimulated with metformin ( $1 \mathrm{mM})$ for 8 hours in a scratch assay. (C) Bar graph showing the gap width $8 \mathrm{~h}$ after scratching (from B). Data are representative of 
means \pm s.e.m. from four independent experiments. Significance of differences between series of results was assessed using one-way analysis of variance, followed by a post-hoc comparison with Dunnett's method for multiple comparisons. $P^{\star * *}<0.001, n=4$. (D) GFP images of AMPK $\alpha 1$-null/KDR/EGFP-WTPdlim5 cells before and after metformin stimulation ( $1 \mathrm{mM})$. Magnified images below show the cells labelled by the number. Scale bar, $10 \square \mathrm{m}$. (E) Western blot of VSMC contractile markers and KLF4 in control, TNF- $\alpha$ and PDGF-BB treated, and metformin combined with those 2 cytokines treated VSMCs.

$\mathbf{A}$
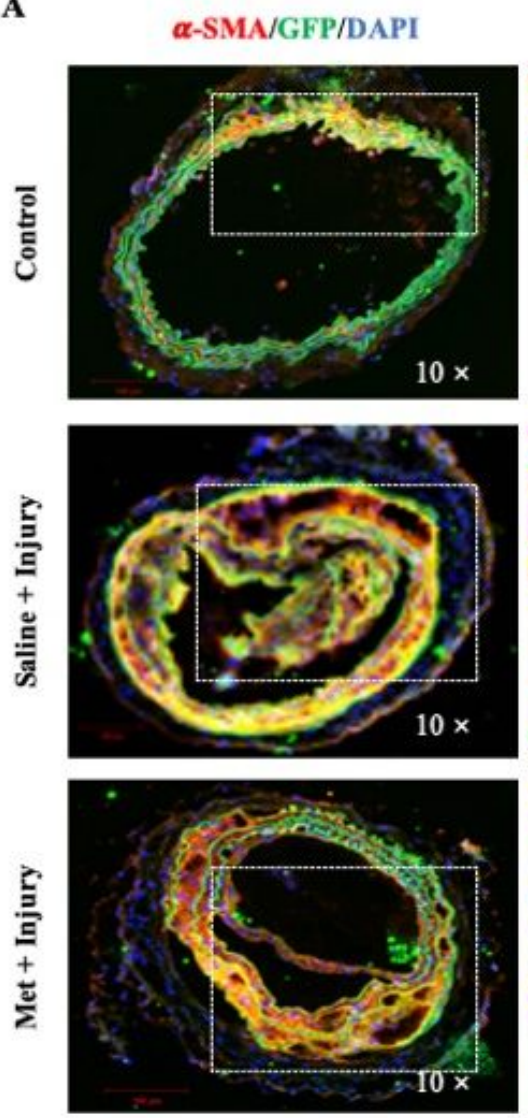

$\alpha$-SMA/GFP/DAPI
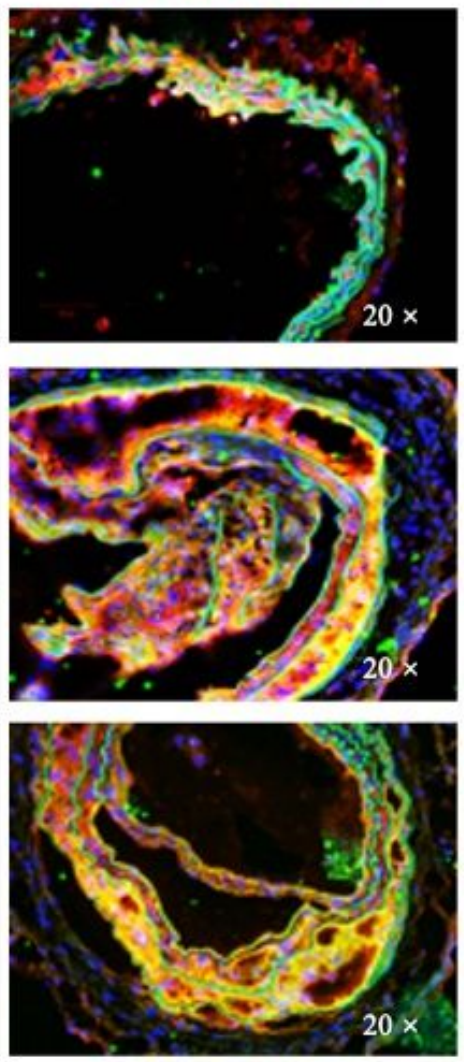

B

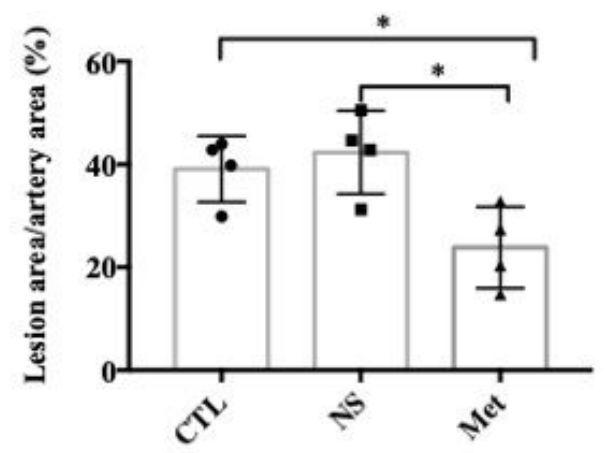

\section{Figure 3}

Metformin alleviated neointima formation in carotid artery injury model. Myh11 cre-eGFP mice were treated with saline or metformin for a week after left carotid wire-injury ( $n=5$ per group). (A) Representative images of immunostaining of SMA and EGFP positive- cells in artery cross section. (B) Quantitative analysis of the percentage of lesion area compared to cross-sectional area of the artery. Data are representative of means \pm s.e.m. from five independent experiments. Significance of differences between series of results was assessed using one-way analysis of variance, followed by a post-hoc comparison with Dunnett's method for multiple comparisons. $P^{*}<0.05, n=5$. 
$\mathbf{A}$
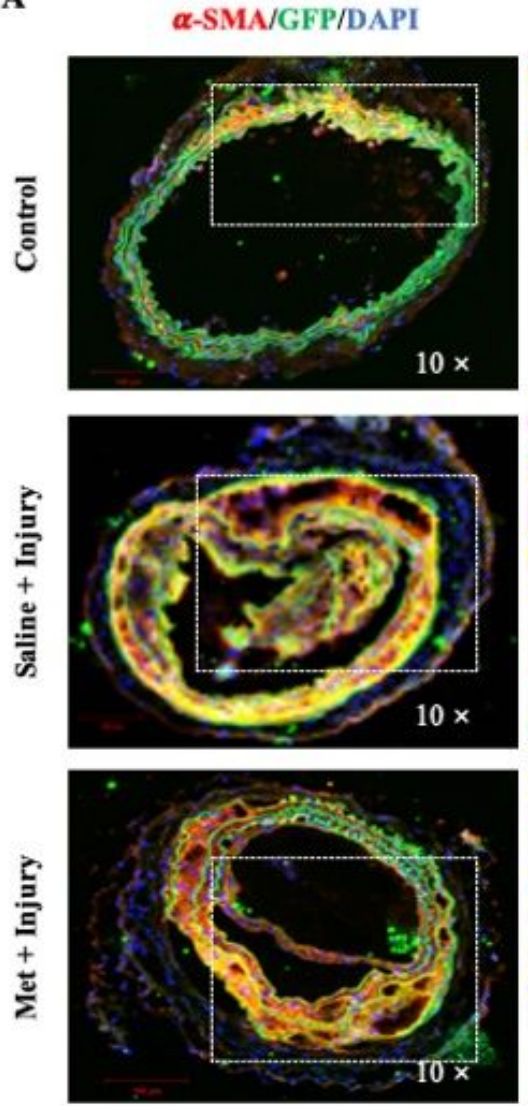

$\alpha$-SMA/GFP/DAPI
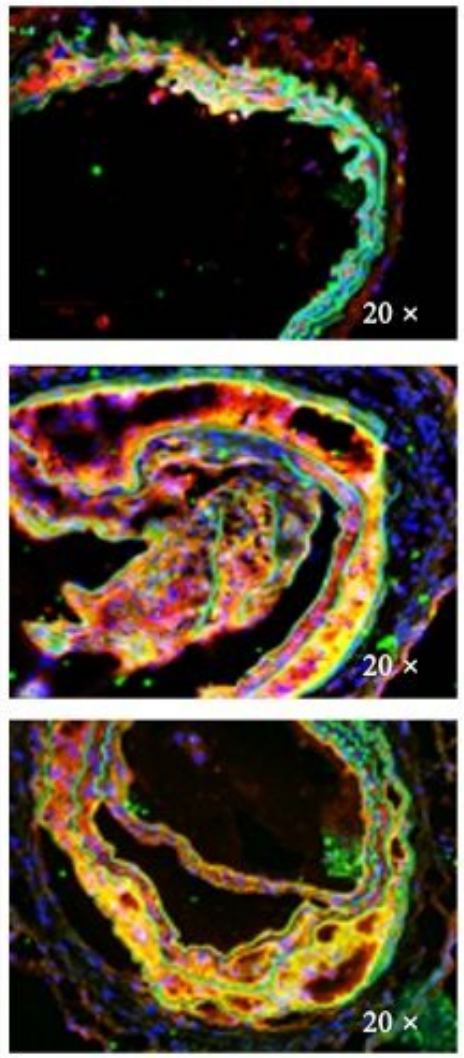

B

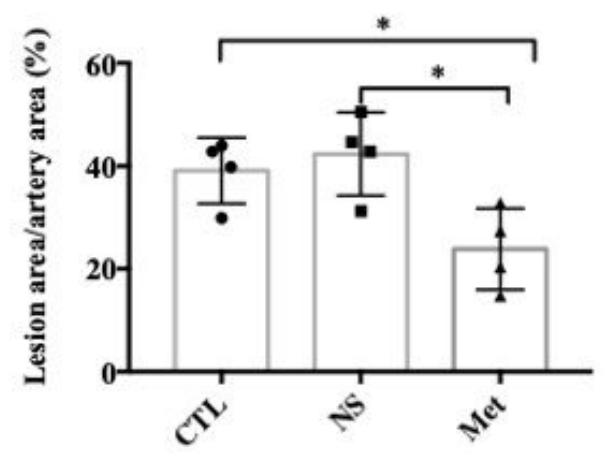

\section{Figure 3}

Metformin alleviated neointima formation in carotid artery injury model. Myh11cre-eGFP mice were treated with saline or metformin for a week after left carotid wire-injury ( $n=5$ per group). (A) Representative images of immunostaining of SMA and EGFP positive- cells in artery cross section. (B) Quantitative analysis of the percentage of lesion area compared to cross-sectional area of the artery. Data are representative of means \pm s.e.m. from five independent experiments. Significance of differences between series of results was assessed using one-way analysis of variance, followed by a post-hoc comparison with Dunnett's method for multiple comparisons. $P^{*}<0.05, n=5$. 
$\mathbf{A}$
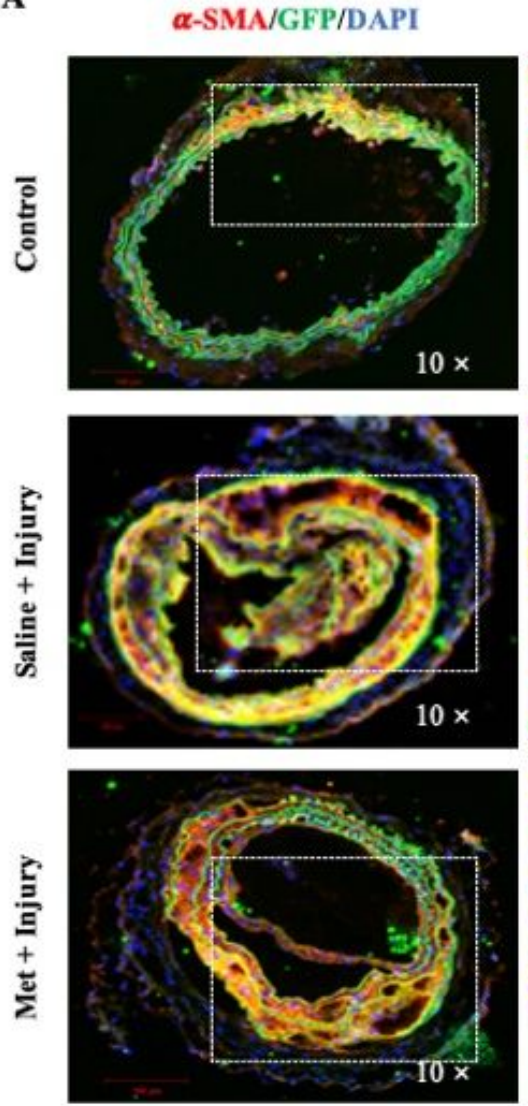

$\alpha$-SMA/GFP/DAPI
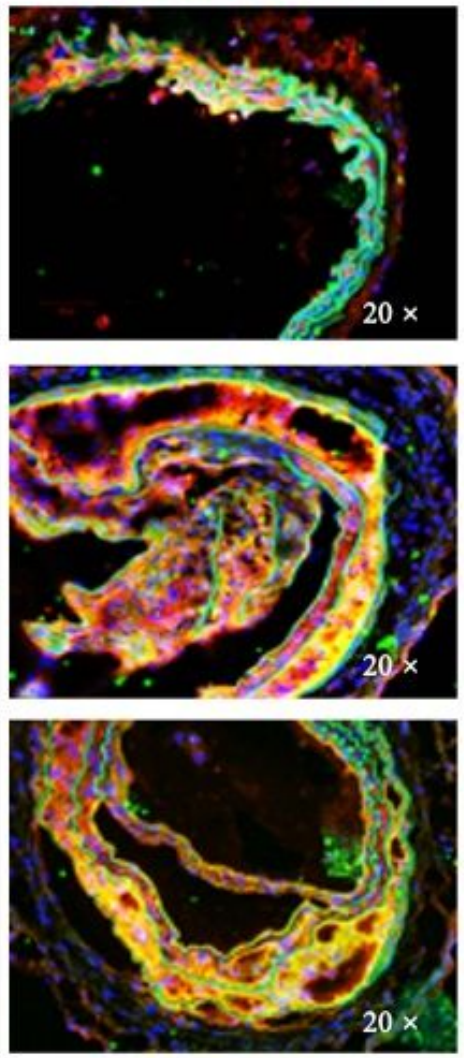

B

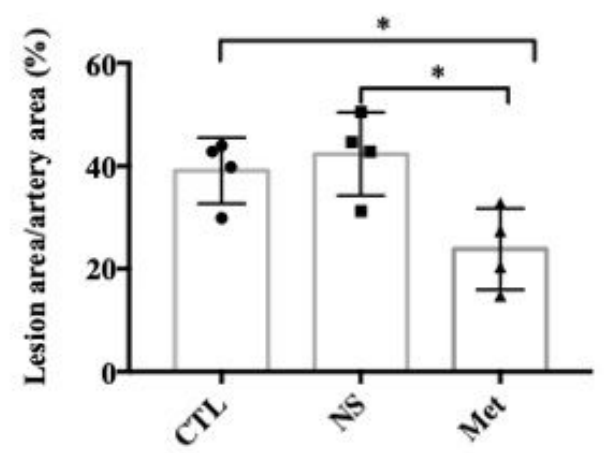

\section{Figure 3}

Metformin alleviated neointima formation in carotid artery injury model. Myh11cre-eGFP mice were treated with saline or metformin for a week after left carotid wire-injury ( $n=5$ per group). (A) Representative images of immunostaining of SMA and EGFP positive- cells in artery cross section. (B) Quantitative analysis of the percentage of lesion area compared to cross-sectional area of the artery. Data are representative of means \pm s.e.m. from five independent experiments. Significance of differences between series of results was assessed using one-way analysis of variance, followed by a post-hoc comparison with Dunnett's method for multiple comparisons. $P^{*}<0.05, n=5$. 
A

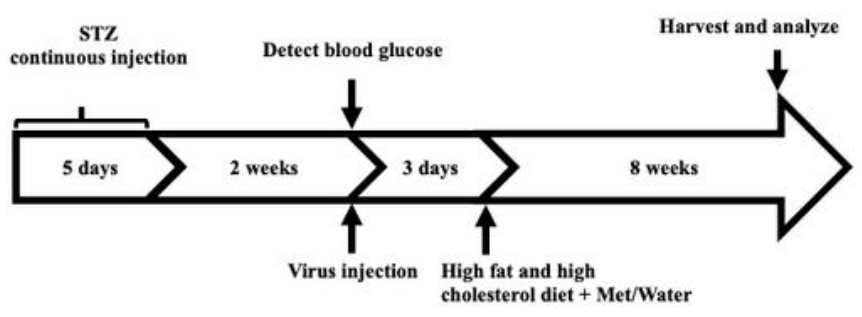

B

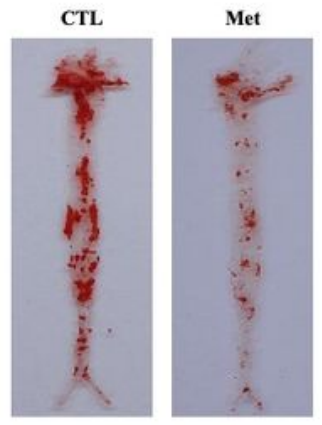

D
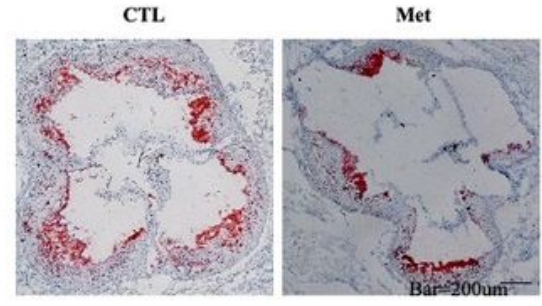

F

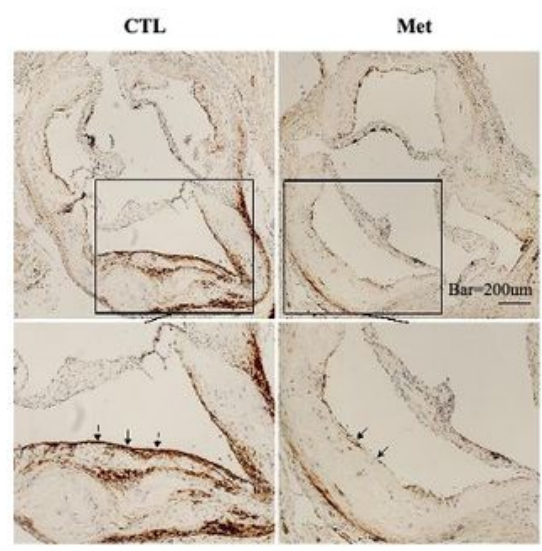

C

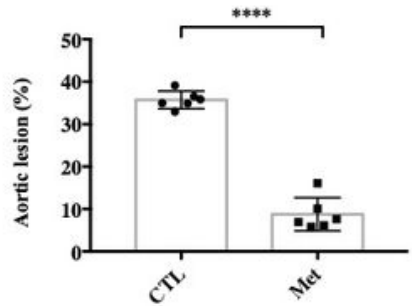

$\mathbf{E}$

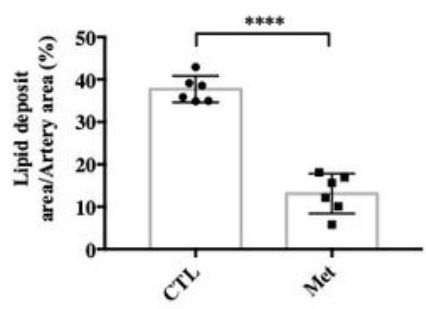

G

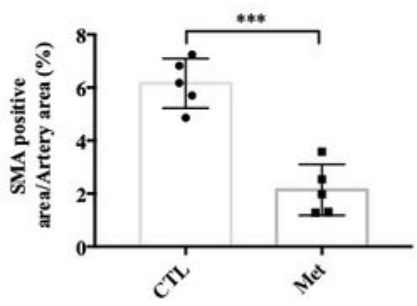

\section{Figure 4}

Metformin inhibited HFD induced atherosclerosis in diabetic ApoE-/- mice. (A) ApoE-/- mice were injected with STZ for 5 days to induce diabetes, then fed with HFD and metformin intervention (Met) or control (CTL) for 8 weeks. (B) Oil Red 0 staining of atherosclerotic lesions at the aorta. (C) Quantification of the en face lesion area in the aorta. Significance of differences was assessed using one-way analysis of variance, followed by a post-hoc comparison with Dunnett's method for multiple comparisons. $\mathrm{P}^{\star \star \star \star}<$ 
$0.0001, n=6$. (D) Oil Red O staining of atherosclerotic lesions at the aortic root. (E) Quantification of the lesion size in the aortic root. Significance of differences was analyzed using one-way analysis of variance, followed by a post-hoc comparison with Dunnett's method for multiple comparisons. $P^{\star \star \star \star}<$

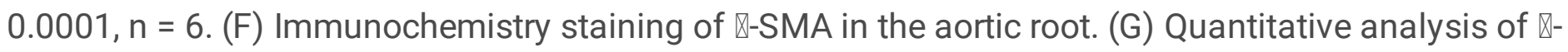
SMA positive area in the aortic root. Significance of differences between series of results was assessed using one-way analysis of variance, followed by a post-hoc comparison with Dunnett's method for multiple comparisons. $P^{\star \star \star}<0.001, n=5$.

A

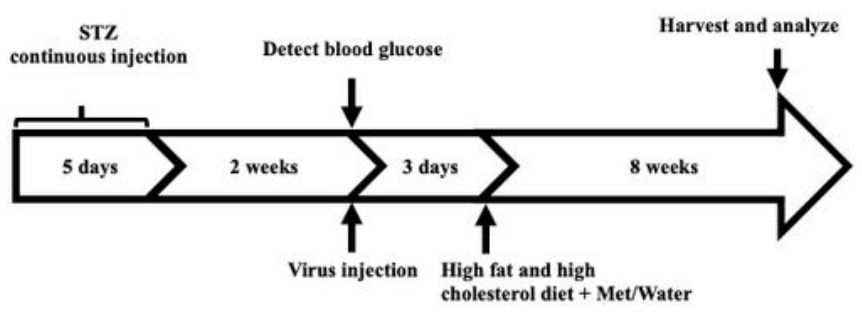

B

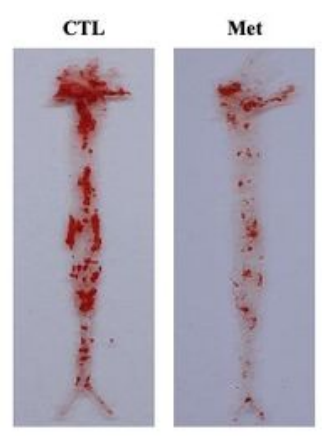

D

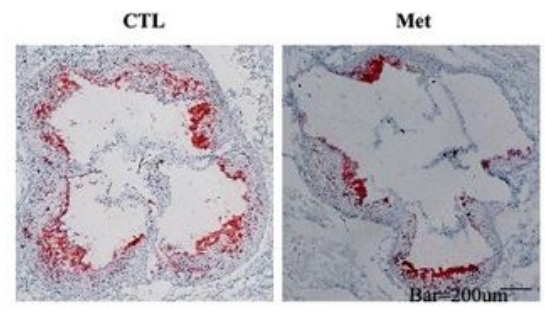

F

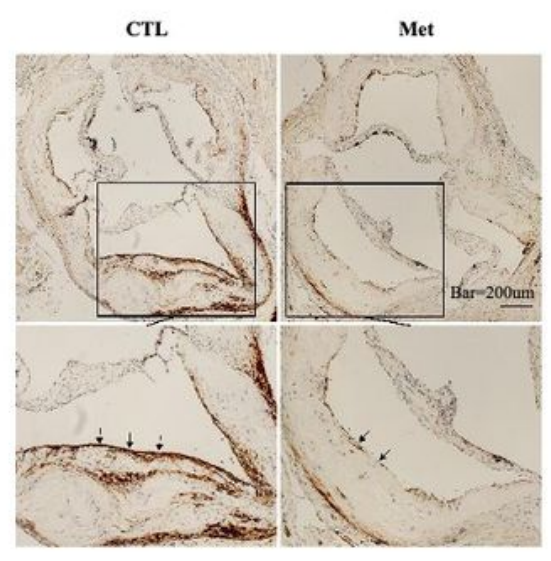

C

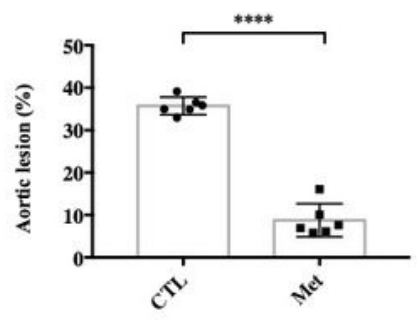

E

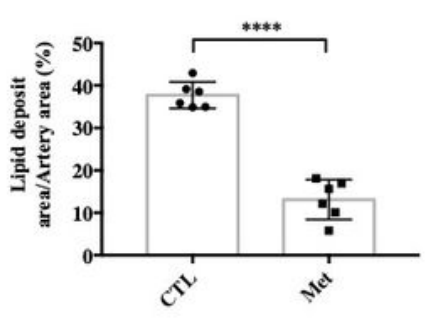

G

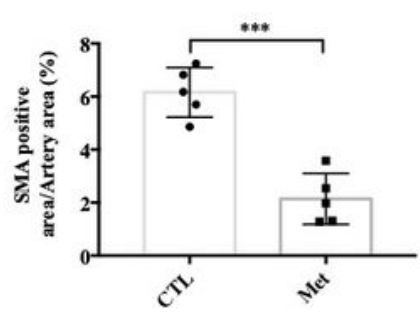




\section{Figure 4}

Metformin inhibited HFD induced atherosclerosis in diabetic ApoE-/- mice. (A) ApoE-/- mice were injected with STZ for 5 days to induce diabetes, then fed with HFD and metformin intervention (Met) or control (CTL) for 8 weeks. (B) Oil Red 0 staining of atherosclerotic lesions at the aorta. (C) Quantification of the en face lesion area in the aorta. Significance of differences was assessed using one-way analysis of variance, followed by a post-hoc comparison with Dunnett's method for multiple comparisons. $P^{\star \star \star \star *}<$ $0.0001, n=6$. (D) Oil Red 0 staining of atherosclerotic lesions at the aortic root. (E) Quantification of the lesion size in the aortic root. Significance of differences was analyzed using one-way analysis of

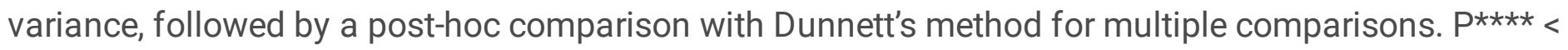

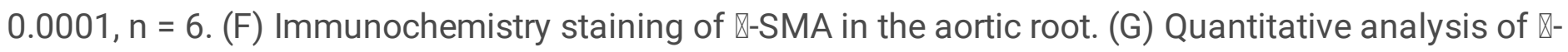
SMA positive area in the aortic root. Significance of differences between series of results was assessed using one-way analysis of variance, followed by a post-hoc comparison with Dunnett's method for multiple comparisons. $P^{\star \star \star}<0.001, n=5$. 
A

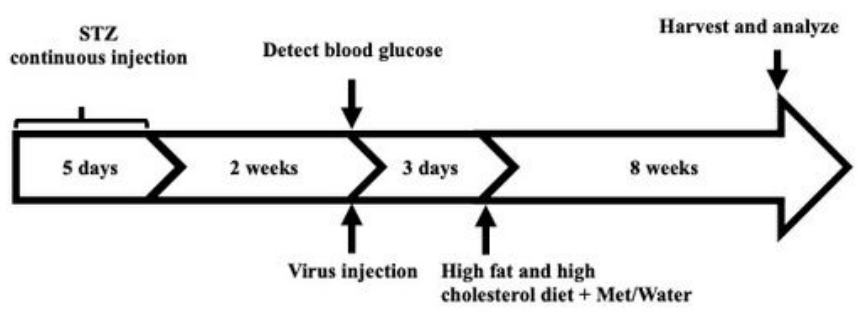

B

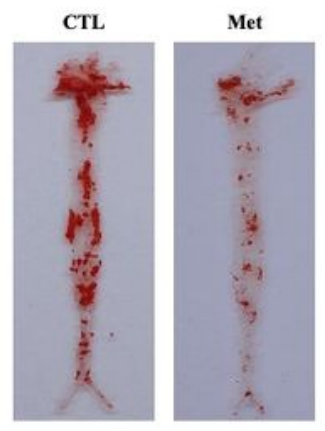

D

CTL
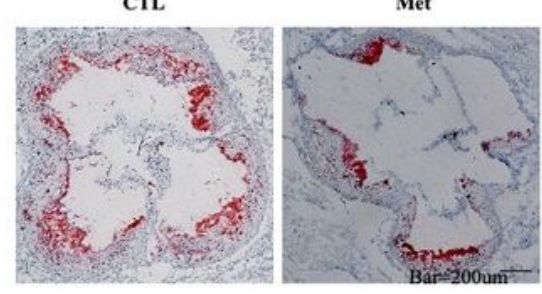

$\mathbf{F}$

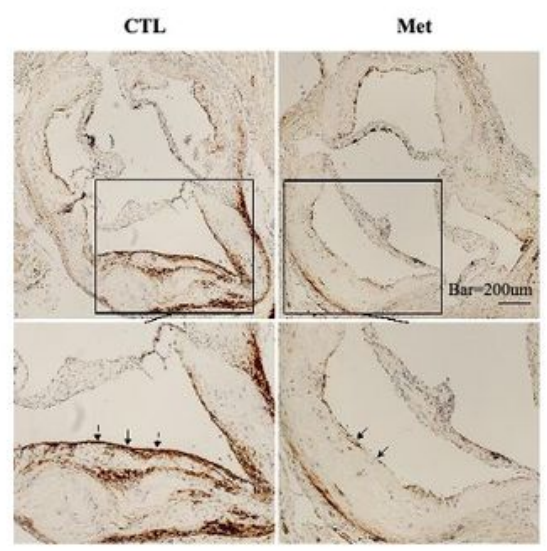

C

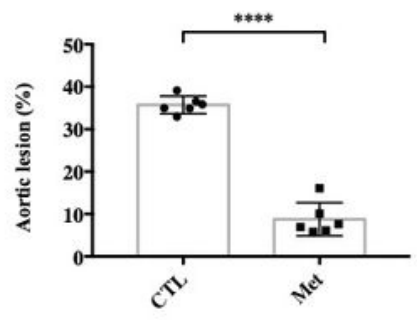

$\mathbf{E}$

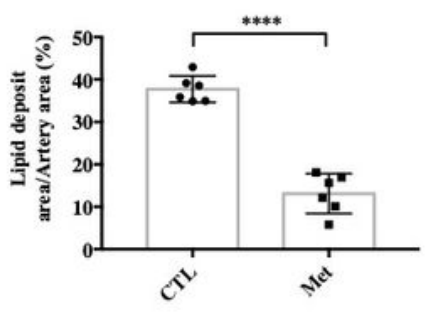

G

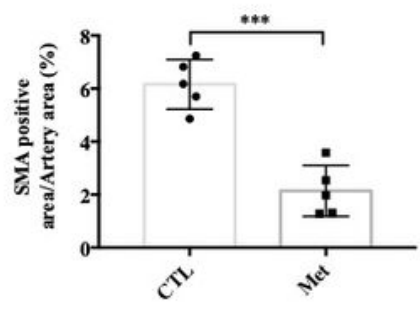

\section{Figure 4}

Metformin inhibited HFD induced atherosclerosis in diabetic ApoE-/- mice. (A) ApoE-/- mice were injected with STZ for 5 days to induce diabetes, then fed with HFD and metformin intervention (Met) or control (CTL) for 8 weeks. (B) Oil Red 0 staining of atherosclerotic lesions at the aorta. (C) Quantification of the en face lesion area in the aorta. Significance of differences was assessed using one-way analysis of variance, followed by a post-hoc comparison with Dunnett's method for multiple comparisons. $\mathrm{P} * \star \star \star<~$ 
$0.0001, n=6$. (D) Oil Red O staining of atherosclerotic lesions at the aortic root. (E) Quantification of the lesion size in the aortic root. Significance of differences was analyzed using one-way analysis of variance, followed by a post-hoc comparison with Dunnett's method for multiple comparisons. $\mathrm{P} * \star \star \star<<$

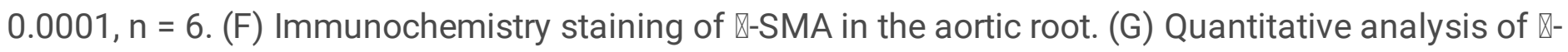
SMA positive area in the aortic root. Significance of differences between series of results was assessed using one-way analysis of variance, followed by a post-hoc comparison with Dunnett's method for multiple comparisons. $P^{\star \star \star}<0.001, n=5$.

$\mathbf{A}$

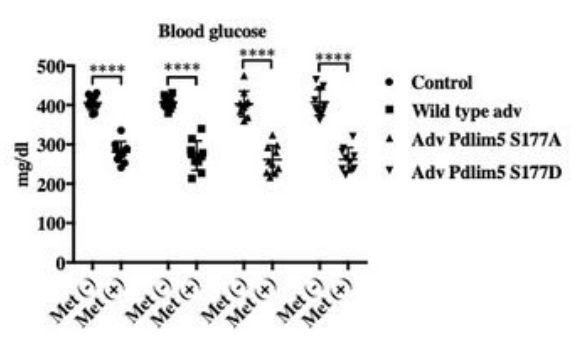

C

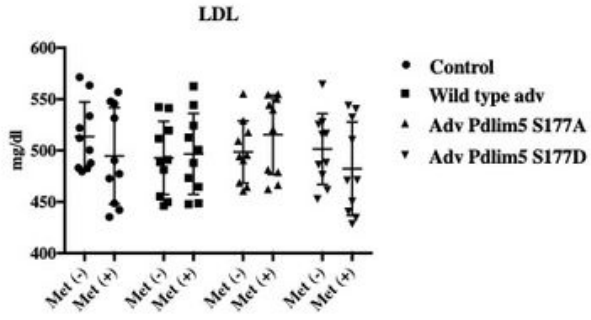

$\mathbf{E}$

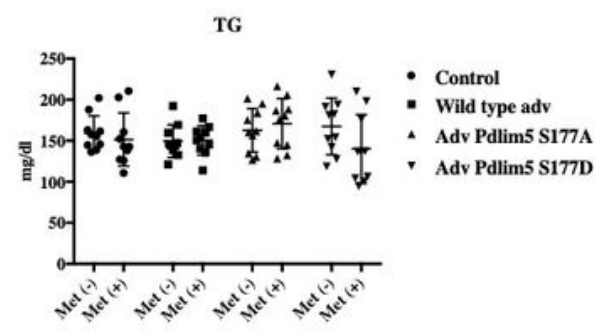

G

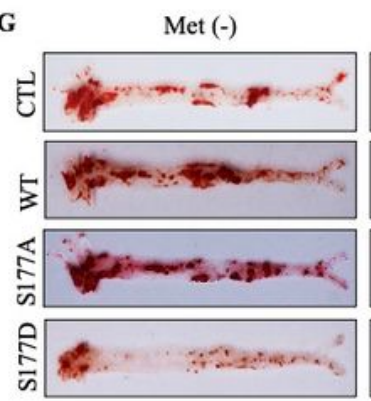

I

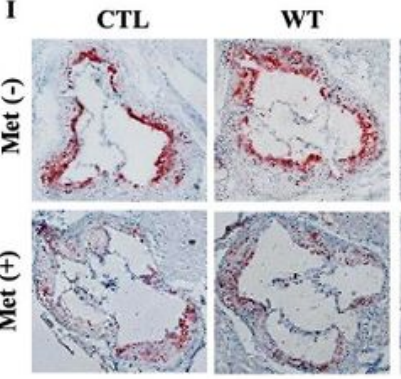

B

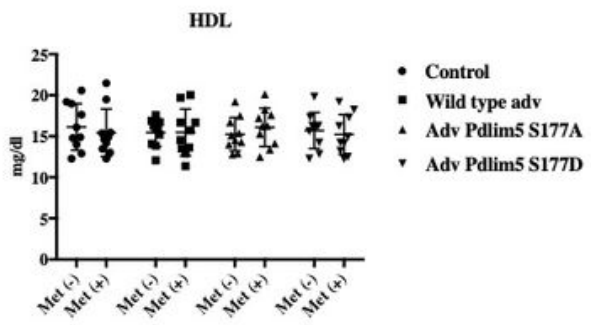

D

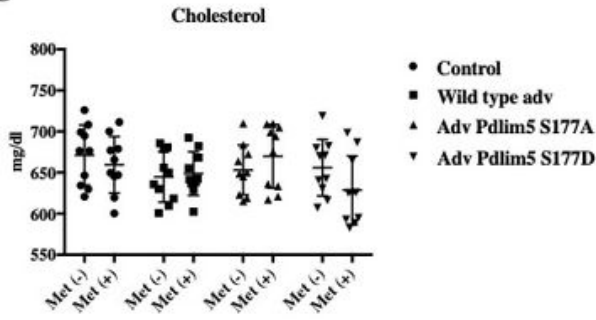

F

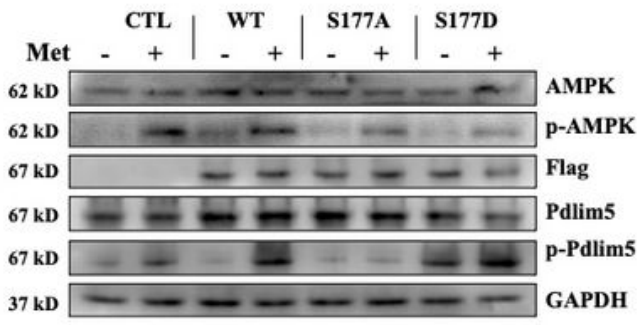

H

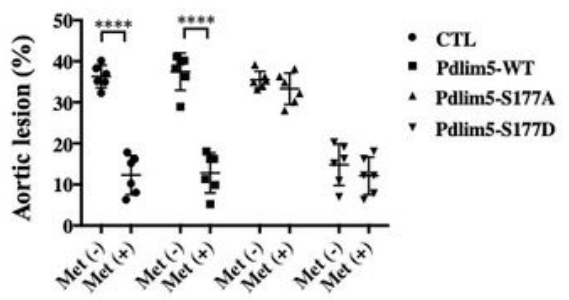

J

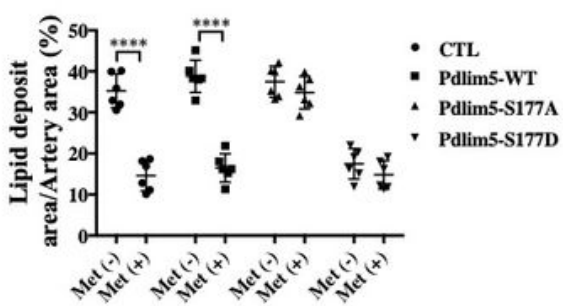




\section{Figure 5}

Phosphorylation of Pdlim5 is involved in the anti-atherosclerosis function of metformin in diabetic ApoE-/- mice. ApoE-/- mice were induced diabetic atherosclerosis and intervened with metformin as described in methods for 8 weeks ( $n=10$ per group). At the same time, mice were randomly separated into 4 groups: Vehicle group, Adenovirus WT Pdlim5 group, Ad S177A Pdlim5 group and Ad S177D Pdlim5 group. (A-E) Blood glucose, plasma HDL, LDL, cholesterol and triglyceride were determined with commercial kit. Data are representative of means \pm s.e.m. from 10 independent experiments. Significance of differences between series of results was assessed using one-way analysis of variance, followed by a post-hoc comparison with Dunnett's method for multiple comparisons. $P * \star \star *<0.0001, n=10$. (F) Metformin induced activation of AMPK and Pdlim5 in aorta were determined with immunoblotting. The lysates of aorta tissues from adenovirus treated mice were collected and subjected to western blotting to determine phosphorylation of AMPK and Pdlim5. (G) Oil Red 0 staining of atherosclerotic lesions at the aorta. $(\mathrm{H})$ Quantification of the en face atherosclerotic lesion area in the aorta. Data are representative of means \pm s.e.m. from six independent experiments. Significance of differences was assessed using oneway analysis of variance, followed by a post-hoc comparison with Dunnett's method for multiple comparisons. $P * \star \star \star<0.0001, n=6$. (I) Oil Red 0 staining of atherosclerotic lesions at the aortic root. (J) Quantification of the atherosclerotic lesion size in the aortic root. Data are representative of means \pm s.e.m. from 6 independent experiments. Significance of differences of results was assessed using one-way analysis of variance, followed by a post-hoc comparison with Dunnett's method for multiple comparisons. $\mathrm{P} \star \star \star \star<0.0001, \mathrm{n}=6$. 
$\mathbf{A}$

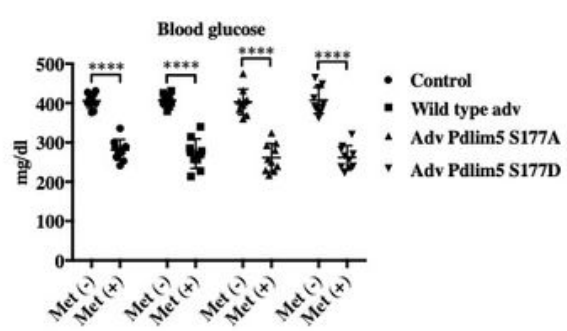

C

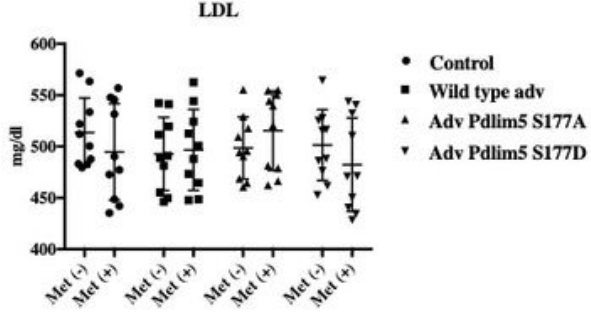

$\mathbf{E}$

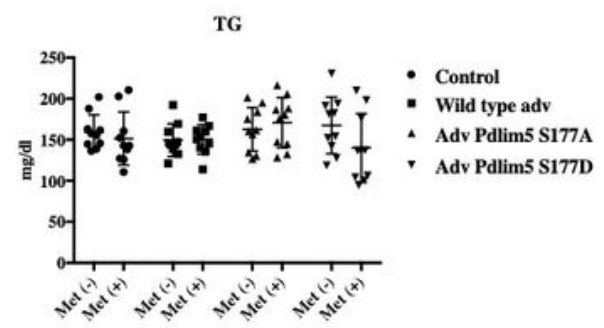

G

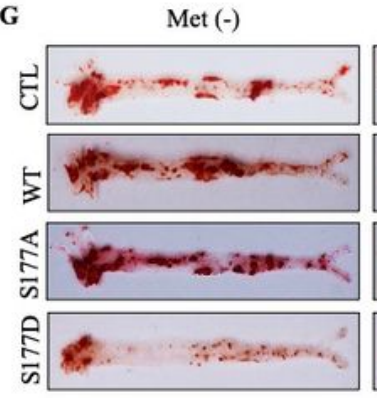

I
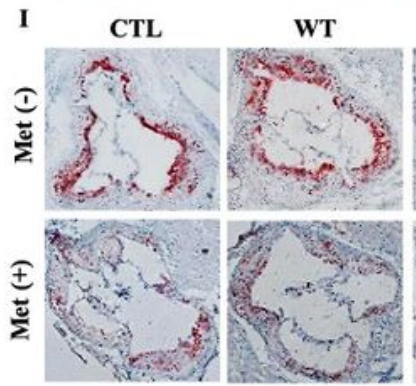

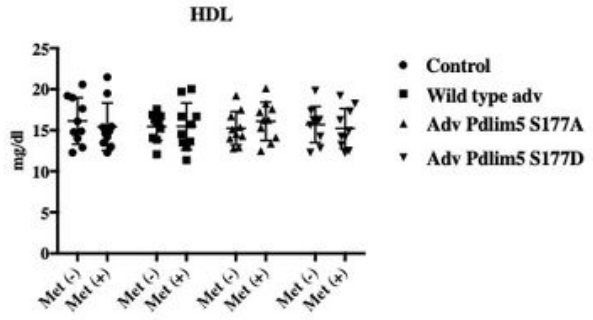

D

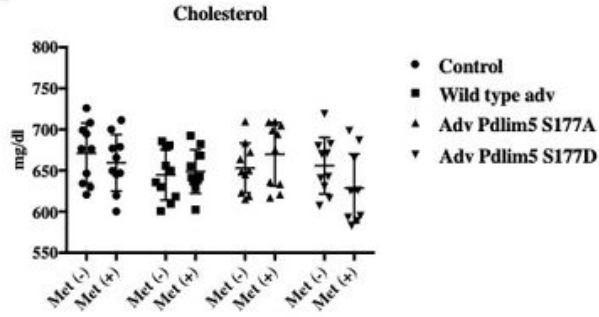

H
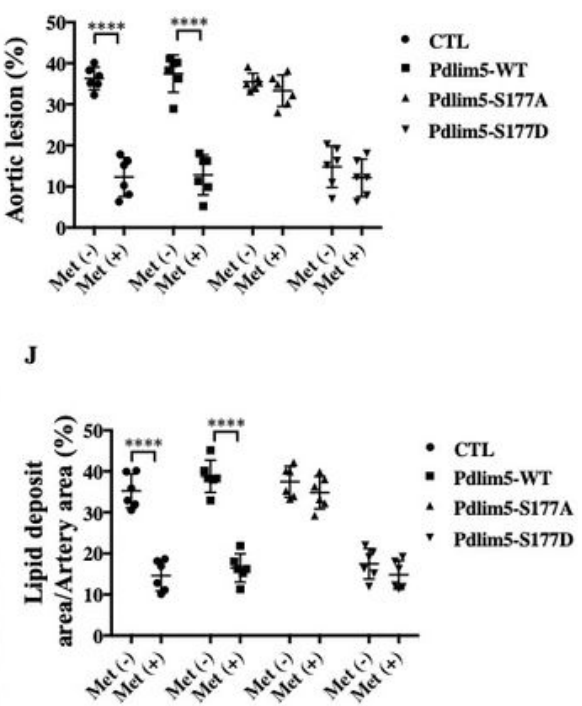

\section{Figure 5}

Phosphorylation of Pdlim5 is involved in the anti-atherosclerosis function of metformin in diabetic ApoE-/- mice. ApoE-/- mice were induced diabetic atherosclerosis and intervened with metformin as described in methods for 8 weeks ( $n=10$ per group). At the same time, mice were randomly separated into 4 groups: Vehicle group, Adenovirus WT Pdlim5 group, Ad S177A Pdlim5 group and Ad S177D Pdlim5 group. (A-E) Blood glucose, plasma HDL, LDL, cholesterol and triglyceride were determined with 
commercial kit. Data are representative of means \pm s.e.m. from 10 independent experiments. Significance of differences between series of results was assessed using one-way analysis of variance, followed by a post-hoc comparison with Dunnett's method for multiple comparisons. $P * \star \star \star<0.0001, n=10$. (F) Metformin induced activation of AMPK and Pdlim5 in aorta were determined with immunoblotting. The lysates of aorta tissues from adenovirus treated mice were collected and subjected to western blotting to determine phosphorylation of AMPK and Pdlim5. (G) Oil Red $O$ staining of atherosclerotic lesions at the aorta. (H) Quantification of the en face atherosclerotic lesion area in the aorta. Data are representative of means \pm s.e.m. from six independent experiments. Significance of differences was assessed using oneway analysis of variance, followed by a post-hoc comparison with Dunnett's method for multiple comparisons. $P * \star \star \star<0.0001, n=6$. (I) Oil Red 0 staining of atherosclerotic lesions at the aortic root. (J) Quantification of the atherosclerotic lesion size in the aortic root. Data are representative of means \pm s.e.m. from 6 independent experiments. Significance of differences of results was assessed using one-way analysis of variance, followed by a post-hoc comparison with Dunnett's method for multiple comparisons. $\mathrm{P} * \star \star \star * 0.0001, \mathrm{n}=6$. 
$\mathbf{A}$

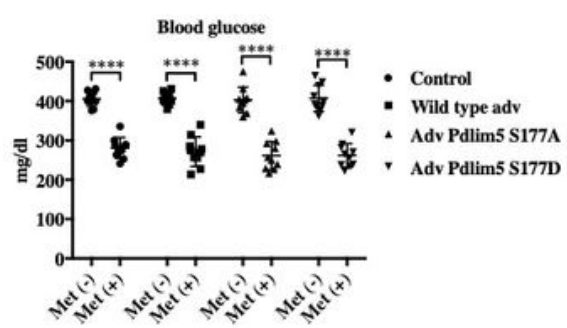

C

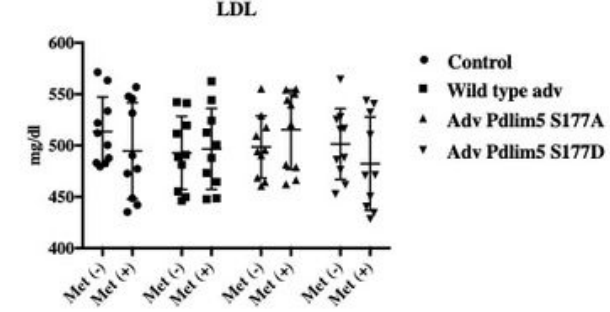

E

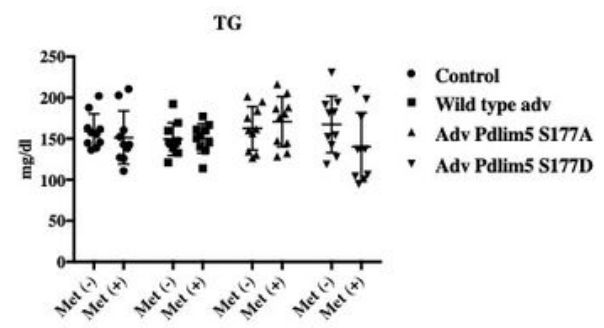

G

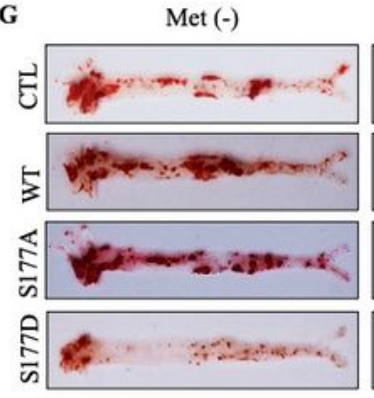

I
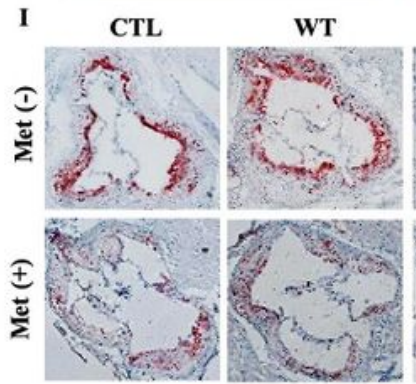

B

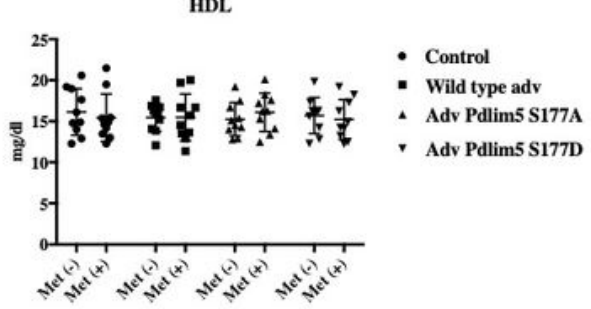

D
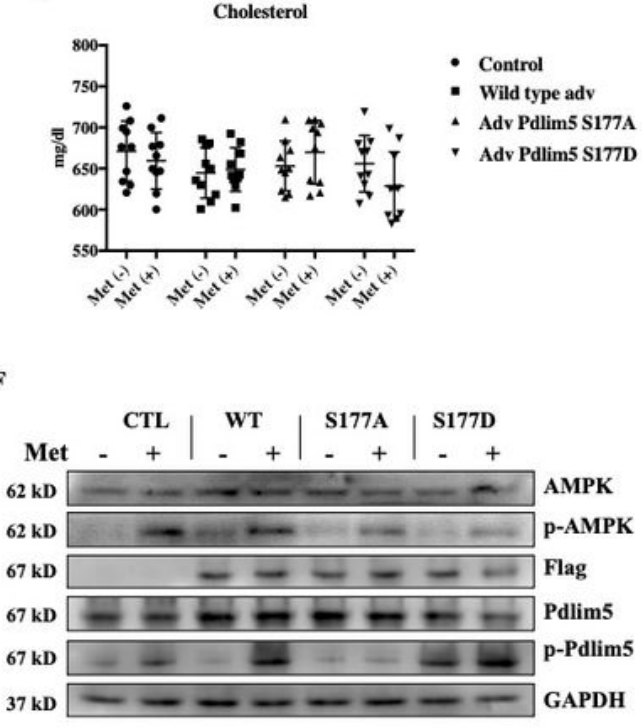

H
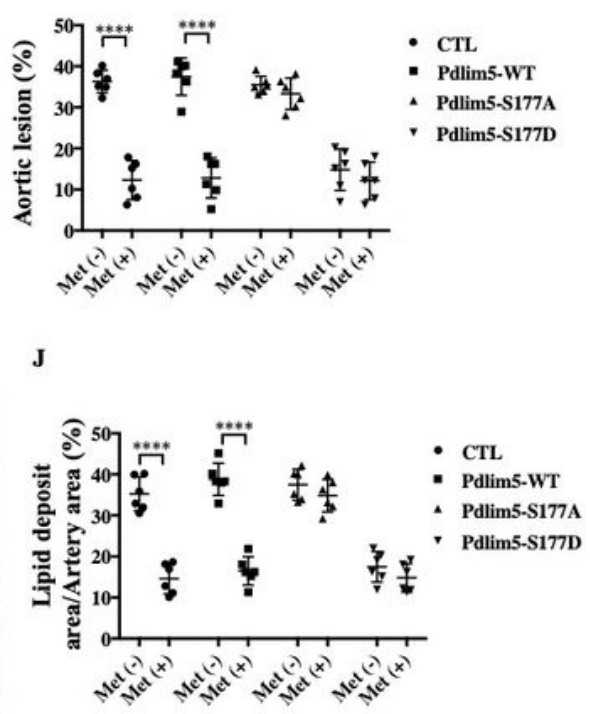

\section{Figure 5}

Phosphorylation of Pdlim5 is involved in the anti-atherosclerosis function of metformin in diabetic ApoE-/- mice. ApoE-/- mice were induced diabetic atherosclerosis and intervened with metformin as described in methods for 8 weeks ( $n=10$ per group). At the same time, mice were randomly separated into 4 groups: Vehicle group, Adenovirus WT Pdlim5 group, Ad S177A Pdlim5 group and Ad S177D Pdlim5 group. (A-E) Blood glucose, plasma HDL, LDL, cholesterol and triglyceride were determined with 
commercial kit. Data are representative of means \pm s.e.m. from 10 independent experiments. Significance of differences between series of results was assessed using one-way analysis of variance, followed by a post-hoc comparison with Dunnett's method for multiple comparisons. $P * \star \star \star<0.0001, n=10$. (F) Metformin induced activation of AMPK and Pdlim5 in aorta were determined with immunoblotting. The lysates of aorta tissues from adenovirus treated mice were collected and subjected to western blotting to determine phosphorylation of AMPK and Pdlim5. (G) Oil Red $O$ staining of atherosclerotic lesions at the aorta. (H) Quantification of the en face atherosclerotic lesion area in the aorta. Data are representative of means \pm s.e.m. from six independent experiments. Significance of differences was assessed using oneway analysis of variance, followed by a post-hoc comparison with Dunnett's method for multiple comparisons. $P * \star \star \star<0.0001, n=6$. (I) Oil Red 0 staining of atherosclerotic lesions at the aortic root. (J) Quantification of the atherosclerotic lesion size in the aortic root. Data are representative of means \pm s.e.m. from 6 independent experiments. Significance of differences of results was assessed using one-way analysis of variance, followed by a post-hoc comparison with Dunnett's method for multiple comparisons. $P * \star * \star<0.0001, n=6$. 

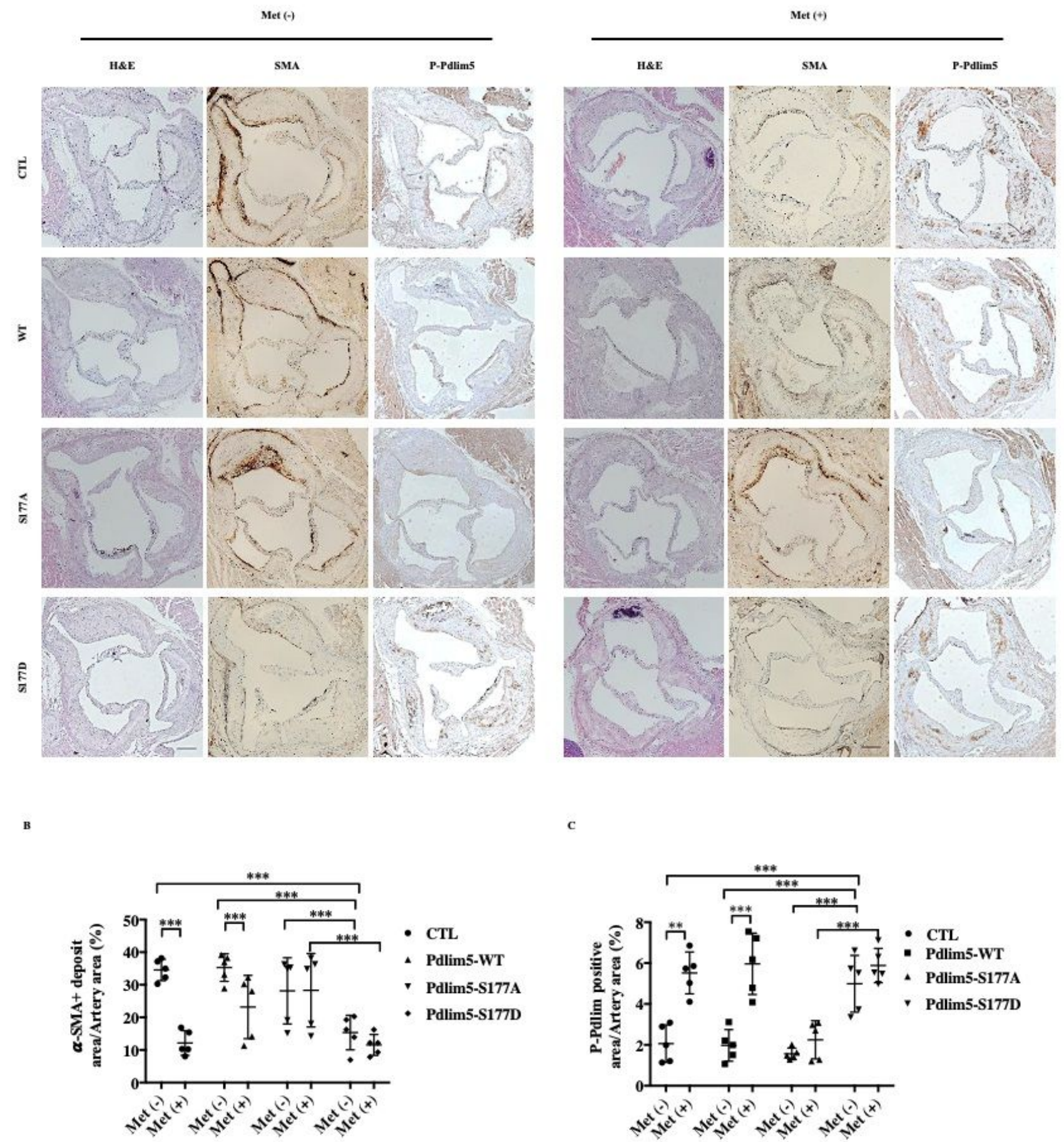

c

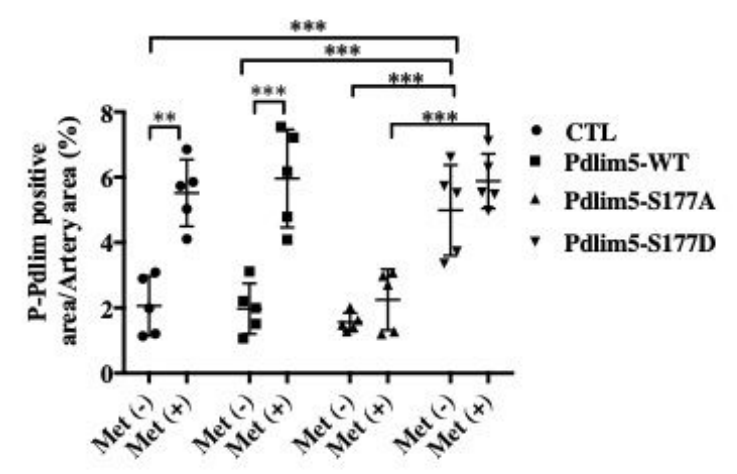

\section{Figure 6}

Phosphorylation of Pdlim5 induced by AMPK was related to metformin's anti-atherosclerosis function. (A)

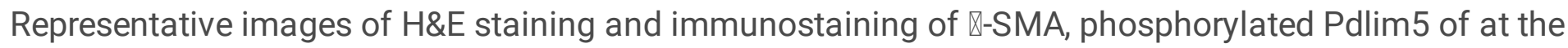
aortic root of diabetic ApoE-/- mice. (B) Quantification of $\mathbb{B}-\mathrm{SMA}$ positive area in the aortic root. Data are representative of means \pm s.e.m. from 5 independent experiments. Significance of differences between series of results was assessed using one-way analysis of variance, followed by a post-hoc comparison 
with Dunnett's method for multiple comparisons. $P^{\star \star \star}<0.001, n=5$. (C) Quantification of phosphorylated Pdlim5 positive area in the aortic root. Data are representative of means \pm s.e.m. from 5 independent experiments. Significance of differences was assessed using one-way analysis of variance, followed by a post-hoc comparison with Dunnett's method for multiple comparisons. $P^{\star \star}<0.01, P^{\star \star \star}<0.001, n=5$.
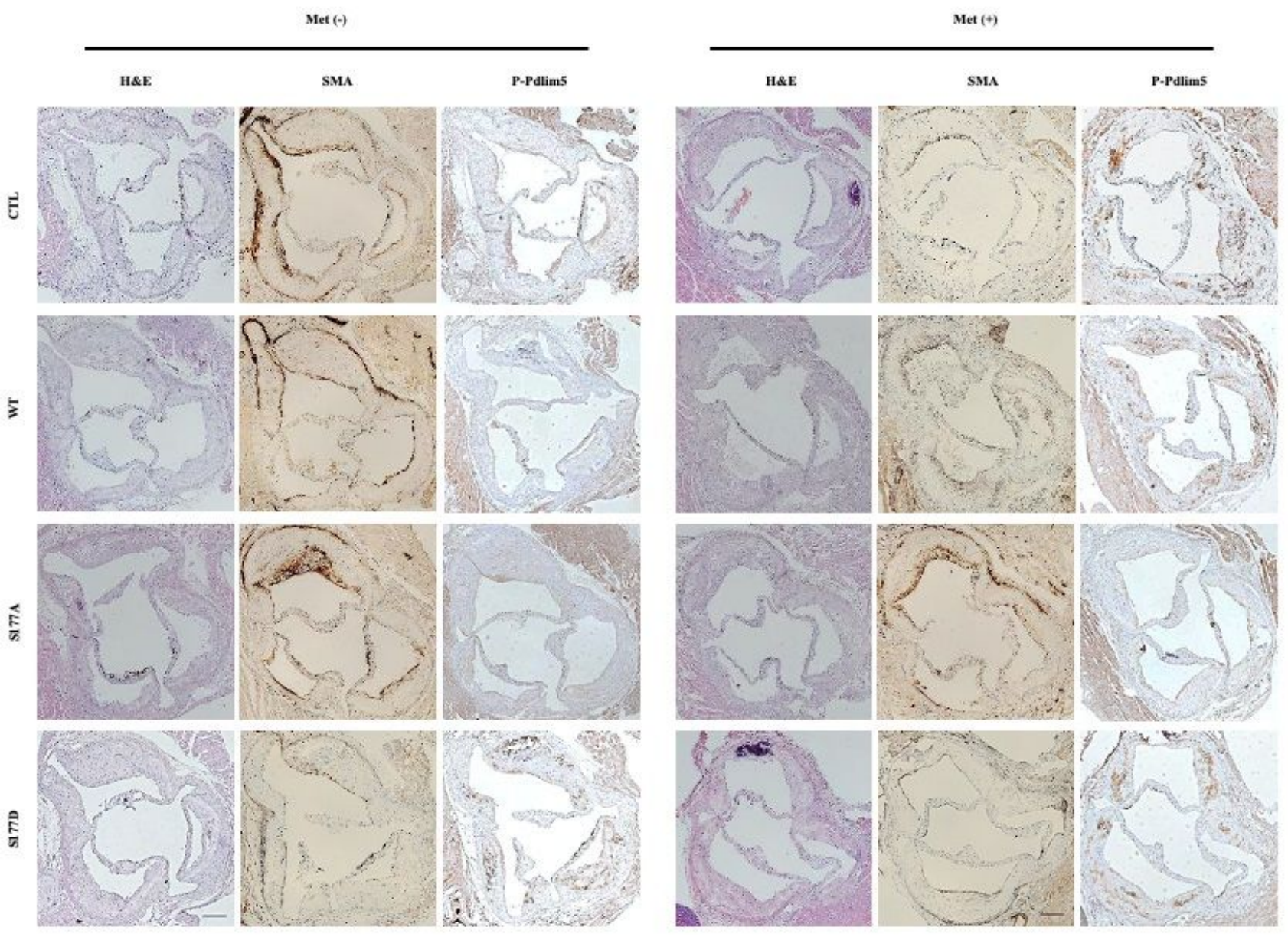

B
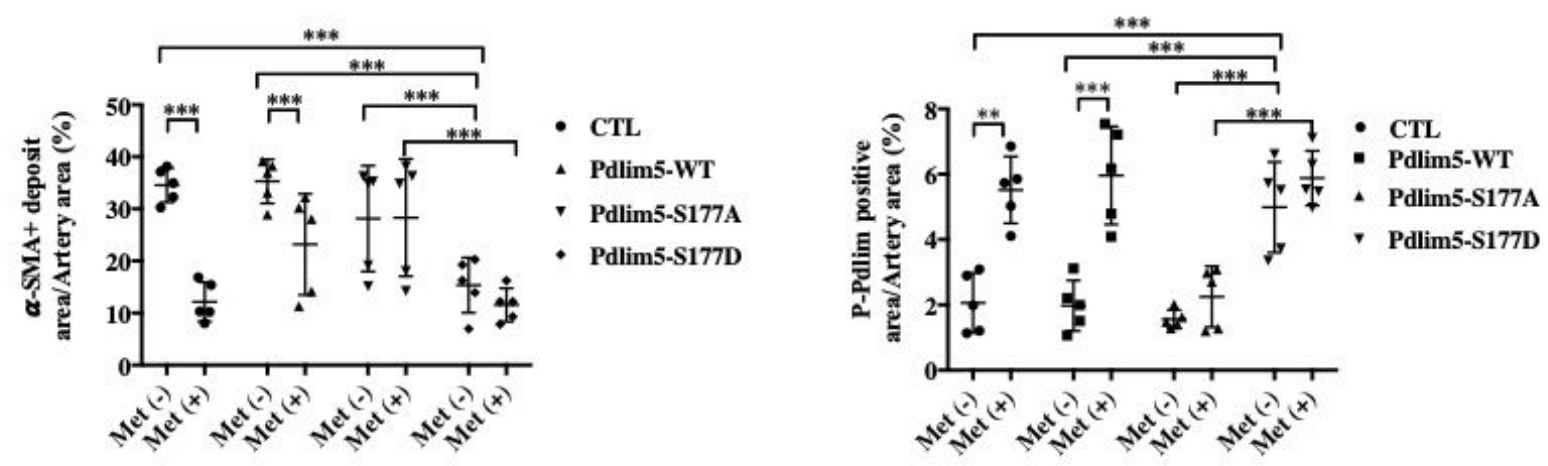

Figure 6 
Phosphorylation of Pdlim5 induced by AMPK was related to metformin's anti-atherosclerosis function. (A)

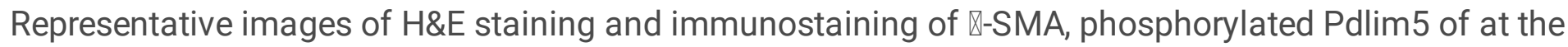
aortic root of diabetic ApoE-/- mice. (B) Quantification of D-SMA positive area in the aortic root. Data are representative of means \pm s.e.m. from 5 independent experiments. Significance of differences between series of results was assessed using one-way analysis of variance, followed by a post-hoc comparison with Dunnett's method for multiple comparisons. $P * \star \star<0.001, n=5$. (C) Quantification of phosphorylated Pdlim5 positive area in the aortic root. Data are representative of means \pm s.e.m. from 5 independent experiments. Significance of differences was assessed using one-way analysis of variance, followed by a post-hoc comparison with Dunnett's method for multiple comparisons. $P \star \star<0.01, P^{\star \star \star}<0.001, n=5$. 

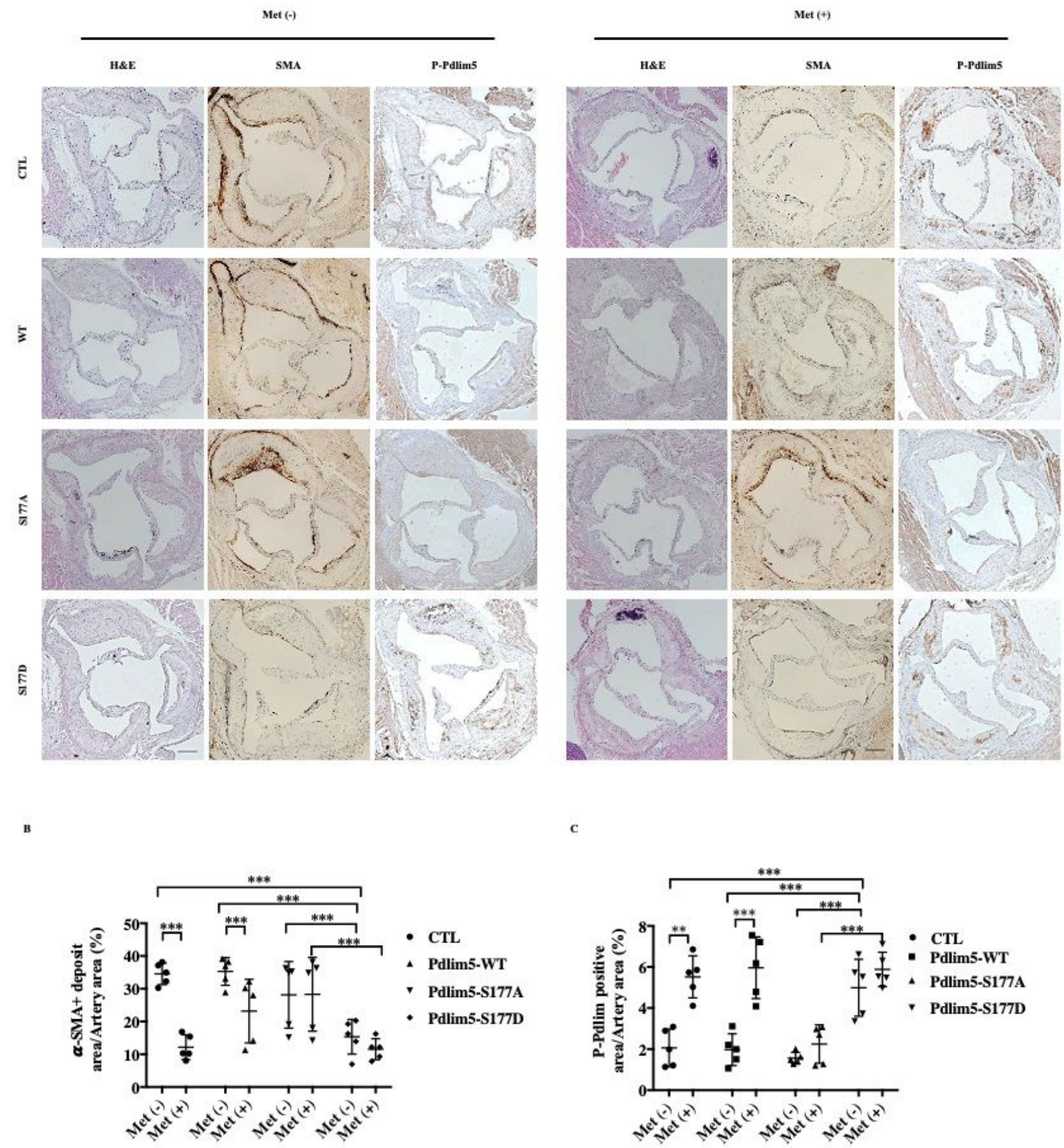

c

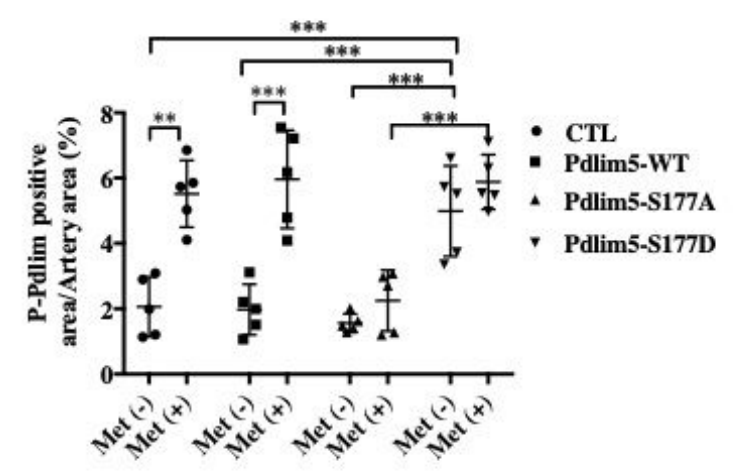

\section{Figure 6}

Phosphorylation of Pdlim5 induced by AMPK was related to metformin's anti-atherosclerosis function. (A)

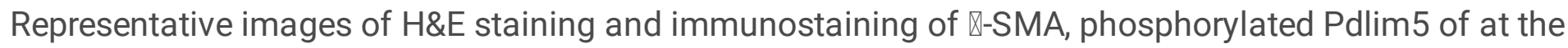
aortic root of diabetic ApoE-/- mice. (B) Quantification of $\mathbb{B}-\mathrm{SMA}$ positive area in the aortic root. Data are representative of means \pm s.e.m. from 5 independent experiments. Significance of differences between series of results was assessed using one-way analysis of variance, followed by a post-hoc comparison 
with Dunnett's method for multiple comparisons. $P^{\star \star \star}<0.001, n=5$. (C) Quantification of phosphorylated Pdlim5 positive area in the aortic root. Data are representative of means \pm s.e.m. from 5 independent experiments. Significance of differences was assessed using one-way analysis of variance, followed by a post-hoc comparison with Dunnett's method for multiple comparisons. $P^{\star \star}<0.01, P^{\star \star \star}<0.001, n=5$. 